\title{
Synthetic Topological Qubits in Conventional Bilayer Quantum Hall Systems
}

\author{
Maissam Barkeshli ${ }^{1}$ and Xiao-Liang Qi ${ }^{2}$ \\ ${ }^{1}$ Station Q, Microsoft Research, Santa Barbara, California 93106-6105, USA \\ ${ }^{2}$ Department of Physics, Stanford University, Stanford, California 94305, USA
}

(Received 6 August 2013; published 20 November 2014)

\begin{abstract}
The idea of topological quantum computation is to build powerful and robust quantum computers with certain macroscopic quantum states of matter called topologically ordered states. These systems have degenerate ground states that can be used as robust "topological qubits" to store and process quantum information. In this paper, we propose a new experimental setup that can realize topological qubits in a simple bilayer fractional quantum Hall system with proper electric gate configurations. Our proposal is accessible with current experimental techniques, involves well-established topological states, and, moreover, can realize a large class of topological qubits, generalizing the Majorana zero modes studied in recent literature to more computationally powerful possibilities. We propose three tunneling and interferometry experiments to detect the existence and nonlocal topological properties of the topological qubits.
\end{abstract}

DOI: 10.1103/PhysRevX.4.041035

\section{INTRODUCTION}

There is currently intense interest in the possibility of realizing topological phases of matter [1,2] that can give rise to non-Abelian quantum statistics [1,3]. When nonAbelian quasiparticles are at fixed, well-separated locations, there is a residual robust degenerate set of states that form a nonlocal Hilbert space protected against any local perturbations. This intrinsic protection against environmental noise has led to proposals to utilize the nonlocal Hilbert space as a "topological qubit" to perform intrinsically robust "topological" quantum computation (TQC) [1].

While non-Abelian statistics was originally proposed in certain exotic non-Abelian fractional quantum Hall (FQH) states $[1,4,5]$, to date it has not been definitively observed experimentally, due to the difficulty and rarity of concrete experimental proposals. There are currently two main directions in the pursuit of non-Abelian statistics: (1) non-Abelian FQH states, such as the famous MooreRead FQH state that may explain the filling fraction 5/2 plateau in GaAs quantum wells $[1,4,6]$, and (2) Majorana fermion zero modes that are bound either to vortex cores in two-dimensional topological superconductors or to the ends of one-dimensional topological superconductors [3,7-13]. A crucial drawback of the Majorana zero modes is their limited computational power, as they cannot be used to create a universal set of gates for TQC.

Remarkably, recent theoretical progress has shown that a wide class of non-Abelian defects can exist, in principle, in

Published by the American Physical Society under the terms of the Creative Commons Attribution 3.0 License. Further distribution of this work must maintain attribution to the author(s) and the published article's title, journal citation, and DOI.
Subject Areas: Condensed Matter Physics,

Quantum Physics

conventional Abelian FQH states, such as the 1/3 Laughlin FQH state [14-18]. These defects have been shown to host "parafermion" zero modes, providing a vast generalization of Majorana fermion zero modes.

In this paper, we show that these abstract ideas are realizable in a concrete, experimentally accessible platform, using conventional Abelian bilayer $\mathrm{FQH}$ states. In contrast to the proposals reviewed above, our setup does not require the realization of exotic new phases matter, and it removes the complication of requiring proximity coupling to superconductivity. Furthermore, the possibility of creating this more general class of defects in $\mathrm{FQH}$ states allows more computationally powerful types of nonAbelian statistics that would be useful for TQC, and paves the way for realizing universal TQC [15]. Our setup (Fig. 1) requires only the simplest bilayer $\mathrm{FQH}$ states that have been observed in the laboratory [19-23], with certain configurations of top and bottom gates. The key idea is to use the gate configuration to induce interlayer tunneling and thus build "staircases" that coherently connect the two layers. The end points of such staircase lines are non-Abelian defects, which we refer to as genons, although the bilayer FQH state itself is Abelian. The non-Abelian statistics of genons are determined by the parent state of the bilayer, which include the Majorana fermion zero modes realized in topological superconductors as a special case.

\section{EXPERIMENTAL SETUP}

Our experimental setup (Fig. 1) consists of a doublelayer quantum well system, with line junctions in each layer that are offset in the lateral direction, induced by top and bottom gates. When the layers are well separated, each layer can individually form its own single-layer FQH state, 
(a)

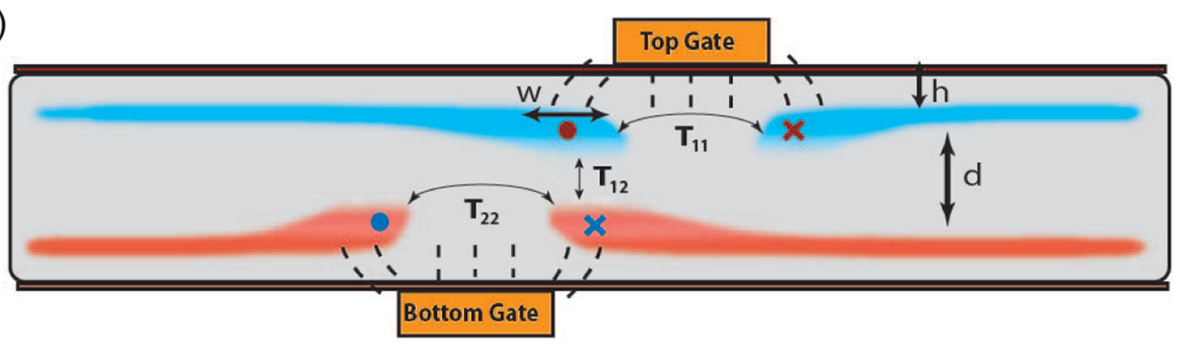

(b)

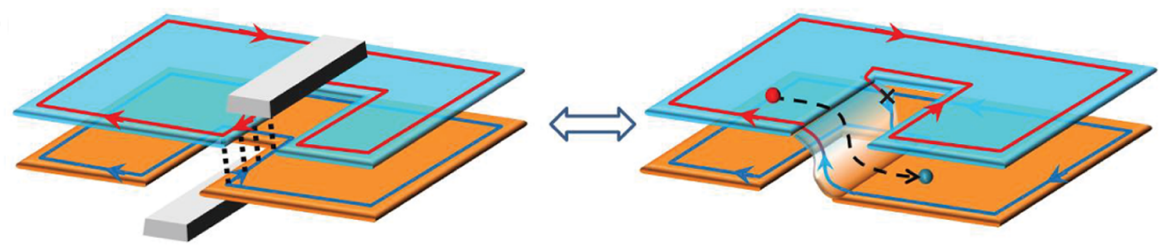

(c)

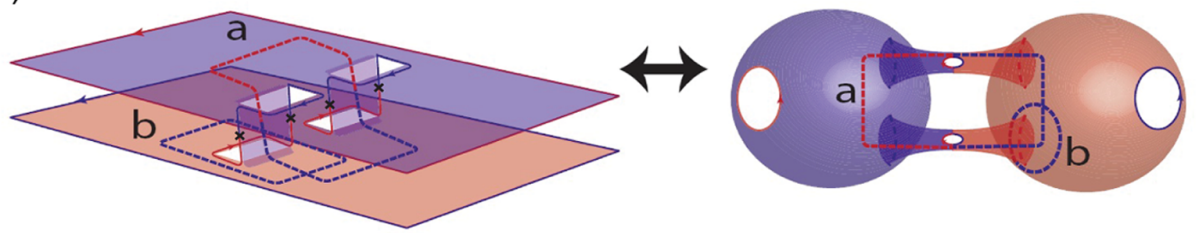

FIG. 1. (a) Cross section of proposed device. Top and bottom gates partially deplete the electron fluids, and the fringe fields cause the wave function at the edges to extend farther vertically, enhancing the tunneling. Filled circles and crosses indicate the gapless chiral edge states, moving out of or into the page. (b) 3D view. Interlayer backscattering between edge states smoothly connects the layers, allowing for coherent quasiparticle propagation between layers. The red and blue balls connected by a dashed line illustrate an interlayer path of the quasiparticle. (c) A pair of TLJs introduces two noncontractible loops, labelled $a$ and $b$, so the space is topologically equivalent to a torus. The edge states around the outer edge of the sample and around the gates are mapped to edge states around four holes on the surface of the torus. In both (b) and (c), the genons (zero modes) are depicted with "x" at the ends of the TLJs.

such as a filling fraction $\nu=1 / m$ Laughlin FQH state. Double-layer 1/3 Laughlin states, which we consider here, have routinely been realized experimentally, with gaps on the order of 5-10 K [19-23].

At the boundary of the line junctions, there are gapless chiral edge states. With the offset between top and bottom gates, and in a suitable parameter region that we analyze below, it is possible to induce backscattering of electrons between counterpropagating edge states from different layers. The backscattering can open a gap at the boundary, such that at long wavelengths quasiparticles can tunnel between the two layers, thus, effectively creating a staircase that coherently connects the layers [Fig. 1(b)]. Such staircases enable the realization of nonplanar geometries. As discussed below, the end points of a staircase line junction are non-Abelian topological defects, a pair of which carries topological degeneracy and thus acts as a topological qubit. We refer to such a staircase as a twisted line junction (TLJ).

\section{A. Conditions for building a TLJ}

The Hamiltonian of the edge states around the line junctions is described by the chiral Luttinger liquid theory [24],

$$
H=\int d x\left(\mathcal{H}_{0}+\delta \mathcal{H}_{t}\right),
$$

with

$$
\begin{aligned}
\mathcal{H}_{0} & =m \pi v_{0} \sum_{\alpha, \beta, I, J} n_{\alpha I} \lambda_{I J}^{\alpha \beta} n_{\beta J}, \\
\delta \mathcal{H}_{t} & =\sum_{I, J} 2\left|T_{I J}\right| \cos \left[m\left(\phi_{L I}+\phi_{R J}\right)+\theta_{I J}\right] .
\end{aligned}
$$

$\mathcal{H}_{0}$ and $\delta \mathcal{H}_{t}$ are the Hamiltonian densities of the kinetic or interaction terms and the backscattering terms, respectively. $\alpha, \beta=L, R$ and $I, J=1,2$ denote the chirality and the layer index of the edge states, respectively. The charge densities on each edge are given by $n_{\alpha I}=(1 / 2 \pi) \partial_{x} \phi_{\alpha I}$, with $\phi_{\alpha I}$ the chiral boson fields satisfying the commutation relations $\left[\phi_{R / L I}(x), \phi_{R / L J}(y)\right]= \pm i(\pi / m) \delta_{I J} \operatorname{sgn}(x-y)$. $T_{I J}=\left|T_{I J}\right| e^{i \theta_{I J}}$ are backscattering amplitudes between counterpropagating edge states $L I$ and $R J$ of the two layers (see Fig. 1). The velocity of the edge modes $v_{0}$ is assumed for simplicity to be the same for all of the edge states. The diagonal entries of $\lambda, \lambda_{I I}^{\alpha \alpha}$ are normalized to 1 , while the off-diagonal elements of the symmetric matrix $\lambda$ parametrize the density-density interaction between the different edge channels. To realize the TLJ, the following conditions are required. 
(1) The line junction of one layer must not destroy the $\mathrm{FQH}$ state directly beneath (or above) it. In the setup of Fig. 1, this means the gates must deplete the mobile electron density in the layer closest to them, while keeping the density in the farther layer such that it remains in its $\mathrm{FQH}$ plateau. Based on previous experimental studies, such a situation is easily possible $[25,26]$.

(2) $T_{12} \gg T_{11}, T_{22}, T_{21}$; i.e., the dominant backscattering term should be $T_{12}$. As long as the width of the line junction $l$ is significantly larger than the interlayer distance $d$, this condition can be easily satisfied. If the system is clean, the intralayer tunneling has a phase factor $T_{I I} \propto$ $e^{i l x / l_{B}^{2}}$ (with $l_{B}=\sqrt{\hbar c / e B}$ the magnetic length) due to the momentum mismatch, while the vertical tunneling has no momentum mismatch. This further suppresses other backscattering terms. Furthermore, the increased potential of the gates induces an exponential enhancement of the interlayer tunneling $T_{12}$ for the edge states in their vicinity, relative to the bulk interlayer tunneling [25,27].

(3) The backscattering $T_{12}$ should generate an energy gap. In the absence of interactions between edge states, the $T_{I J}$ are irrelevant perturbations in the renormalization group sense, and a large tunneling $T_{12} \gtrsim \hbar v_{0} / l_{B}$ is required to open an energy gap and lead to the required TLJ. However, it is possible for the screened Coulomb interaction between edge states to make $T_{12}$ a relevant perturbation, so that any small $T_{12}$ will open a gap. We estimate $m \pi \hbar v_{0} \lambda_{I J}^{\alpha \beta} / l_{B} \sim U_{I J}^{\alpha \beta}$, where $U_{I J}^{\alpha \beta}$ is the Coulomb energy between edges. When the gates are wide $(l \gg d)$, the only appreciable interaction will be between the two edges on top of each other, which, ignoring screening due to the gates, is estimated by $m \pi \hbar v_{0} \lambda_{12}^{R L} / l_{B} \sim e^{2} / \epsilon d$. In this case, the scaling dimension of the electron backscattering term is $\Delta_{t}=m \sqrt{\left(1-\lambda_{12}^{R L}\right) /\left(1+\lambda_{12}^{R L}\right)}$ [28]. The tunneling is relevant in the renormalization group sense if $\Delta_{t}<2$ in the clean limit, or $\Delta_{t}<3 / 2$ for disorder-induced backscattering (assuming Gaussian-correlated disorder). For realistic parameters in GaAs samples, $v_{0} \sim 10^{5} \mathrm{~m} / \mathrm{s}, d \sim 100 \mathrm{~nm}$, $l_{B} \sim 10 \mathrm{~nm}, \epsilon \sim 13 \epsilon_{0}$, we see that the repulsive interaction can easily make $\lambda_{12}^{R L}$ to be order 1 , so that $T_{12}$ will be relevant.

We note that it would be advantageous to make the width of the edge region $w$ [Fig. 1(a)] over which the density gradually decreases to zero to be as small as possible (ideally $w \sim l_{B}$ ). For this reason, in addition to GaAs double quantum wells, two layers of graphene separated by a thin insulating barrier may be an advantageous system [27]. If instead $w \gg l_{B}$, disorder in the TLJ will help localize any additional gapless modes that may appear due to edge reconstruction. In GaAs systems, we expect $h \sim w \sim 50 \mathrm{~nm}$ to be accessible, and $d \sim 100 \mathrm{~nm}$.

Now that we have analyzed the feasibility of building the TLJ, we discuss the realization of topological qubits and experimental predictions.

\section{TOPOLOGICAL DEGENERACY: GEOMETRICAL PICTURE}

A $\nu=1 / m$ Laughlin FQH state on a genus $g$ surface has a ground-state degeneracy $m^{g}$ [29]. In our experimental proposal, with two TLJs [Fig. 1(c)], the system now has two independent noncontractible loops $a$ and $b$ that intersect only once; the system is topologically equivalent to a torus with four holes cut out on the surface [Fig. 1(c)]. This topological degeneracy is directly related to the fractional statistics of the quasiparticles, which imply the following magnetic algebra for the quasiparticle loop operators $\hat{W}(a)$ and $\hat{W}(b): \hat{W}(a) \hat{W}(b)=e^{2 \pi i / m} \hat{W}(b) \hat{W}(a) . \hat{W}(a)$ and $\hat{W}(b)$ measure the fractional charge inside the loop $a, b$, respectively. The ground states form an irreducible representation of this algebra, which is at least $m$ dimensional. With $n$ TLJs, the system is topologically equivalent to a genus $g=n-1$ surface, with $2 n+2$ holes cut out on the surface, which will have a topological degeneracy of $m^{g}$. On a finite size system, quasiparticle-quasihole tunneling around $a, b$ mixes the different states, splitting the degeneracy by an energy that is at most exponentially small in system size.

The holes cut on the surface contribute chiral edge states with a finite size gap inversely proportional to the perimeter of the hole, but they do not affect the topological degeneracies. Nevertheless, we note that the gapless edge states surrounding the TLJs can be removed in two ways. First, while the bare value of $T_{21} \approx 0$, higher-order tunneling processes will generate $T_{21} \sim T_{11} T_{22} / T_{21}$, perturbatively in $T_{I I} / T_{21}$. Thus, both pairs of edge states can be gapped, in principle. Alternatively, they can also be removed through more complicated interdigitated gate configurations (see Appendix B).

Since each TLJ increases the degeneracy by a factor of $m$, each end point of the TLJ, viewed as a point defect, can be associated with $\sqrt{m}$ degrees of freedom. Similar to the Majorana zero mode carrying $\sqrt{2}$ degrees of freedom, each end point of a TLJ is a non-Abelian topological defect, which we refer to as a genon [15]. The resulting topological degeneracies can be used to realize a topological qubit; this setup enables manipulations of such topological qubits by braiding the genons. The braiding process can be shown to correspond to topologically nontrivial surgery operations of the genus $g$ surface [15]. These mathematical operations correspond to cutting the space along a noncontractible loop, rotating by $2 \pi$, and gluing back together. Physically, these braiding operations can be implemented within our experimental setup by manipulating the gate configuration, as shown in Appendix E.

\section{PROPOSED EXPERIMENTS}

\section{A. Interlayer fractional quantized Hall conductance}

The $\sqrt{m}$ topological degeneracy associated with a genon, i.e., an end point of the TLJ, corresponds to a localized parafermion zero mode [14-18,30,31], which 
generalizes the usual Majorana zero modes in topological superconductors. Zero-mode operators $\alpha_{i}$ can be defined at each genon, which satisfies the parafermion algebra $\alpha_{i} \alpha_{j}=\alpha_{j} \alpha_{i} e^{2 \pi i / m}, i<j$, with a proper ordering of all genons in the system. Physically, the operator $\alpha_{i}$ is a quasiparticle tunneling operator between the two layers at the genon point, which commutes with the Hamiltonian. We refer the reader to Ref. [15] and Appendix A for a more precise definition of the zero-mode operators.

In the case of the Majorana zero modes localized at the ends of a 1D topological superconductor, one of its key signatures is a quantized zero-bias conductance peak with conductance $G=2 e^{2} / h$ when a normal metal lead is coupled to the Majorana zero mode $[3,32,33]$. This is due to the perfect Andreev reflection in the presence of the Majorana mode. In our system, the analog of an electron in a topological superconductor is a quasiparticle-quasihole pair, i.e., a topological exciton with charge $(1 / m,-1 / m)$. Here, the two numbers represent the charge of the exciton in the two layers, in units of the electron charge $e$. When such an exciton moves across the TLJ, the two layers are exchanged, such that its charge becomes $(-1 / m, 1 / m)$; a topological exciton will become its antiparticle upon crossing a TLJ, just like an electron is transformed into a hole in a superconductor through an Andreev process. The topological exciton has a perfect resonant Andreev reflection at zero energy in the presence of the parafermion zero mode, which can be probed experimentally [Fig. 2(a)].

By separately contacting the two layers, as shown in Fig. 2(a), the edge states can carry different currents, and the relative current $I_{r}=I_{1}-I_{2}$ is the exciton current. If we switch-off the TLJ (by turning off the gate voltages), the two layers are decoupled and the voltage in each layer is proportional to its current. Therefore, $d I_{r} / d V_{r}=$ $(1 / m)\left(e^{2} / h\right)$. With the TLJ, the two layers are exchanged when the edge states meet the TLJ, so that the two voltages are also reversed, and $d I_{r} / d V_{r}=-(1 / m)\left(e^{2} / h\right)$. This interlayer fractional quantized Hall conductance is a unique signature of the parafermion zero modes, directly analogous to the zero-bias peak in Majorana wires.

\section{B. Exponential localization of zero mode through virtual STM}

To further verify that the zero-bias-peak conductance observed from the perfect Andreev reflection experiment is really from a local parafermion zero mode, we further consider the experimental setting in Fig. 2(b), which is an analog of using a STM to probe the Majorana zero mode [9]. By contacting source and drain to the two different layers and bringing the edge states close to the TLJ, the edge states carry the exciton current $I_{r}$ and play the role of a STM tip. It is well known that the electron tunneling from a STM tip leads to a differential conductance proportional to the local electron density of states. By analogy, the tunneling conductance $d I_{r} / d V_{r}$ in Fig. 2(b) measures the
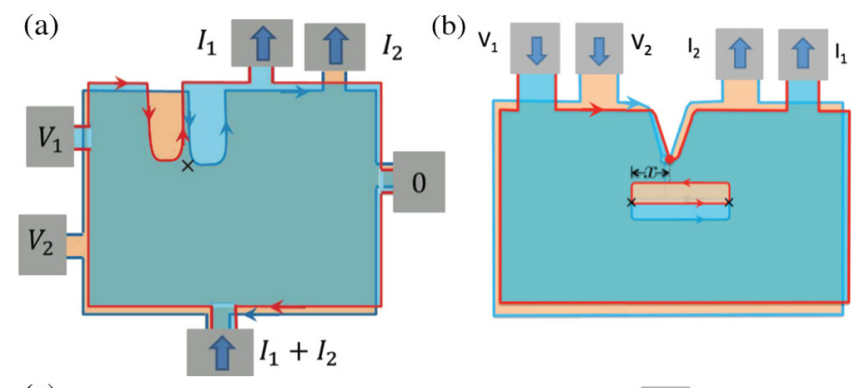

(c)

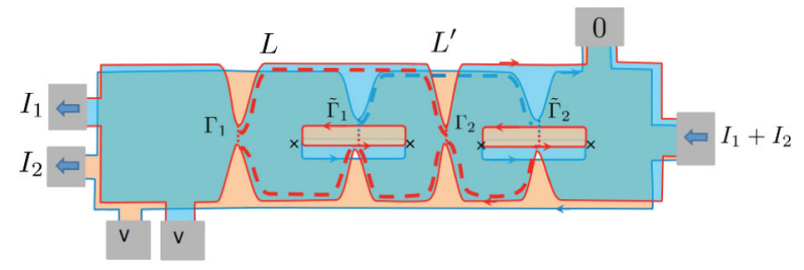

FIG. 2. Experimental setups to measure the non-trivial properties of the genons/parafermion zero modes. (a) Experimental setup to measure the interlayer fractional quantized Hall conductance, where the exciton current, $I_{r}=I_{1}-I_{2}$, is reversed as it encounters the TLJ. The quantization at zero temperature and frequencies demonstrates that this is a resonant process closely analogous to the resonant Andreev reflection for Majorana zero modes in topological superconductors. (b) The outer edge states can be used to measure the local quasiparticle density of states, through the interlayer conductance $d I_{r} / d V_{r}$, thus detecting the exponential localization of the parafermion zero modes at the ends of the TLJ. (c) QPC interferometer setup for detecting the non-Abelian nature of the genons. $\Gamma_{1,2}$ are the amplitudes for quasiparticle tunneling across the two QPCs in the first layer, and $\Gamma_{1,2}$ are the amplitudes for quasiparticle tunneling through the two interlayer QPCs across the TLJs. $L$ and $L^{\prime}$, indicated by the thick dashed lines, are the two interference loops.

local density of states of the exciton, which is set by the correlation function

$$
G_{(1,-1)}(x, \omega)=\left\langle V_{(1,-1)}^{\dagger}(x, \omega) V_{(1,-1)}(x,-\omega)\right\rangle .
$$

$V_{(1,-1)}(x, t)=e^{i \phi_{L 1}(x, t)-i \phi_{L 2}(x, t)}$ annihilates an exciton in the upper edge of the TLJ, or equivalently, tunnels a $1 / m$ quasiparticle from layer 2 to layer 1 . We find $G_{(1,-1)}$ has the following exponential behavior:

$$
G_{(1,-1)}(x, \omega)=f(x) e^{-|x| / \xi} \delta(\omega),
$$

where $\xi \sim \hbar v / g$ is the correlation length of the gapped region (with $g$ the gap opened at the TLJ), and $f(x)$ is a power-law function of $x$. The exponential localization, despite the presence of the gapless edge excitations, is a remarkable result. As we show in Appendix A, it physically occurs because, away from the ends of the TLJ, $V_{(1,-1)}$ necessarily creates a gapped solitonic excitation; at the ends of the TLJ, $V_{(1,-1)}$ keeps the system in the ground-state manifold and yields a unitary transformation among the different ground states. Therefore, the correlations of $V_{(1,-1)}$ must decay exponentially away from the ends of 
the TLJ. From this analysis, we see that the zero-bias peak in the exciton local density of states is indeed probing the topologically degenerate states.

\section{Long-time current-noise cross-correlation}

Since the genons are non-Abelian defects, it should be possible to detect them through interferometry. Compared to the interferometry measurements proposed for detecting non-Abelian anyons in non-Abelian FQH states [6,34,35], an advantage of our current setup is that the non-Abelian defects (genons) are imposed externally rather than being dynamical quasiparticle excitations, so that their position and number can be easily controlled.

Our setup [Fig. 2(c)] consists of two TLJs and four quantum point contacts (QPCs), created by either a top gate or a bottom gate. One pair of QPCs $\left[\Gamma_{1,2}\right.$ in Fig. 2(c) $]$ allows for weak quasiparticle tunneling across the normal region of the fluid, between the edges of the top layer. The other pair $\left(\tilde{\Gamma}_{1,2}\right)$ allows for weak quasiparticle tunneling across the TLJs, from one edge of the top layer to the other edge of the bottom layer. There are only two nontrivial interference loops formed by the four QPCs: the first one [ $L$ in Fig. 2(c)] in the top layer including $\Gamma_{1}$ and $\Gamma_{2}$, and the second one $\left(L^{\prime}\right)$ going through both layers including $\tilde{\Gamma}_{1}$ and $\tilde{\Gamma}_{2}$. The interference in these two loops $L, L^{\prime}$ measures the electric charge in the loops, which measures the topological qubit state of the genons 1,2 and 2,3, respectively. As a key consequence of the non-Abelian nature of genons, the topological qubit degrees of freedom are nonlocal, and the topological charge carried by genons 1,2 does not commute with that of 2,3 . Therefore, nonlocal correlations between interference patterns in these two loops are induced due to the uncertainty principle. Alternatively, the noncommutativity of the two quasiparticle paths $L$ and $L^{\prime}$ can also be understood from the geometrical picture shown in Fig. 1(c), with the measured topological charge corresponding to $\hat{W}(a), \hat{W}(b)$.

The interference pattern can be observed by applying a voltage difference $V$ between the left- and right-moving edges in both layers and measuring the currents $I_{1}, I_{2}$ [Fig. 2(c)]. We find that the noise correlation between layers

$$
S_{12}(t) \equiv \frac{1}{2}\left\langle\left\{I_{1}(t), I_{2}(0)\right\}\right\rangle-\left\langle I_{1}(t)\right\rangle\left\langle I_{2}(0)\right\rangle
$$

has a dramatic feature:

$$
S_{12}(|t| \gg 1 / T) \rightarrow \text { const } \neq 0 .
$$

Such a long-time noise correlation, at finite temperatures, is a striking direct consequence of the non-Abelian nature of the genons. The current $I_{1}(t)$ depends on the quasiparticle interference between the two QPCs $\Gamma_{1,2}$. When a quasiparticle leaves the top layer through the QPC $\tilde{\Gamma}_{1}$, its fractional statistics cause a phase shift in the interference of loop $L$ and, therefore, changes the value of $I_{1}(t)$. Such a phase shift is permanent until the next quasiparticle tunneling occurs at $\tilde{\Gamma}_{1}$.
The converse is also true, that the quasiparticle tunneling at $\Gamma_{2}$ causes a permanent phase shift in $I_{2}(t)$. Therefore, the fluctuation of the currents $I_{1}(t)$ and $I_{2}(0)$ has a long-time correlation for both $t>0$ and $t<0$. It should be noted that having only three QPCs [36], $\Gamma_{1}, \tilde{\Gamma}_{1}, \Gamma_{2}$ can already induce a long-time correlation of $S_{12}$, but only for $t>0$.

In Appendix C, we present an explicit calculation of $S_{12}$. The result, for $|t| \gg 1 / T$ and to lowest order in the tunneling strength at the QPCs, takes the form

$$
\begin{aligned}
S_{12}(t)= & T^{(4 / m)-2}\left\{\left|\Gamma_{1}^{2} \tilde{\Gamma}_{1} \tilde{\Gamma}_{2}\right| \theta(-t) S_{12}^{(1)}+\left|\tilde{\Gamma}_{1}^{2} \Gamma_{1} \Gamma_{2}\right| \theta(t) S_{12}^{(2)}\right. \\
& \left.+\left|\Gamma_{1} \Gamma_{2} \tilde{\Gamma}_{1} \tilde{\Gamma}_{2}\right|\left[S_{12}^{(3)} \theta(t)+S_{12}^{(4)} \theta(-t)\right]\right\} .
\end{aligned}
$$

The dimensionless functions $S_{12}^{(i)}$, whose explicit form is given in the Appendix D, depend on the quasiparticle statistics (they are nonzero only when $m \neq 1$ ) and decay with the dimensionless ratios $\hbar e V / m k_{B} T$ and $k_{B} T x / \hbar v$, which are optimally of order 1, where $x$ is the distance scale between the QPCs. Note $S_{12}^{(i)} \rightarrow 0$ at high temperatures when phase coherence is lost, as expected. Also note that thermally excited mobile quasiparticle can decohere the state of the qubit on a time scale exponentially long in $1 / T$.

\section{DISCUSSION}

A longer-term goal is to implement unitary gates on the topological qubit by braiding genons (see Appendix E). This can be realized with further sets of gates to tune interactions between various zero modes localized at the genons $[15,16,17,37]$. The computational power of genons in generic Abelian states is stronger than that provided by Majorana zero modes [16]. The setup proposed here can be generalized to realize universal TQC in non-Abelian states that by themselves would not be universal for TQC [15]. Examples include two layers of the non-Abelian phase of Kitaev's honeycomb model $[15,38]$ or the odd-denominator Bonderson-Slingerland FQH states [39,40].

\section{ACKNOWLEDGMENTS}

We thank David Goldhaber-Gordon, Woo-Won Kang, Charlie Marcus, and Hari Manoharan for helpful conversations. This work was supported by the Simons Foundation (M. B.) and David and Lucile Packard foundation (X.-L. Q.).

\section{APPENDIX A: PARAFERMION ZERO MODES}

As mentioned in the main text, an important property of the TLJs is that their ends localize exotic forms of zero modes, called parafermion zero modes, which generalize the usual Majorana zero-mode algebra $[14,15]$. Here, we briefly recall the definition and physical meaning of these zero-mode operators, reviewing results from Refs. [14,15].

Let us imagine aligning all TLJs, and cutting the whole system along the line, yielding the counterpropagating edge states. Then, the normal regions of the fluid can be obtained 
by gluing the edges back together with the electron tunneling terms $\sum_{I}\left(\Psi_{e L I}^{\dagger} \Psi_{e R I}+\right.$ H.c. $) \propto \sum_{I} \cos \left(m \phi_{I}\right)$, while the twisted line junctions can be described by gluing the edges back together with the twisted tunneling $\Psi_{e L 1}^{\dagger} \Psi_{e R 2}+\Psi_{e L 2}^{\dagger} \Psi_{e R 1}+$ H.c. $\propto \sum_{I} \cos \left(m \tilde{\phi}_{I}\right)$, where we have defined $\phi_{I}=\phi_{L I}+\phi_{R I}$ and $\tilde{\phi}_{I}=\phi_{L I}+\phi_{R(I+1) \% 2}$. Here, we also include the tunneling term $\Psi_{e L 2}^{\dagger} \Psi_{e R 1}+$ H.c., which is not present in the experimental setup of Fig. 1.

The ends of the TLJ can, therefore, be considered as domain walls between these two different ways of gapping the counterpropagating edge states:

$$
\delta \mathcal{H}_{\text {tunn }}=g \begin{cases}\sum_{I} \cos \left(m \phi_{I}\right) & \text { if } x \in A_{i} \\ \sum_{I} \cos \left(m \tilde{\phi}_{I}\right) & \text { if } x \in B_{i},\end{cases}
$$

where $A_{i}$ indicates the normal regions, while $B_{i}$ are the regions associated with the TLJs.

As shown in Refs. [14,15], we can define quasiparticle tunneling operators (see Fig. 3) $\alpha_{2 i-1}=e^{i \phi_{1}\left(x_{A_{i}}\right)} e^{-i \tilde{\phi}_{1}\left(x_{B_{i}}\right)}$, $\alpha_{2 i}=e^{i \tilde{\phi}_{2}\left(x_{B_{i}}\right)} e^{-i \phi_{2}\left(x_{A_{i+1}}\right)}$, which are zero modes of the edge Hamiltonian $\left[\alpha_{i}, H_{\text {edge }}\right]=0$, and which satisfy a $Z_{m}$ parafermion algebra, $\alpha_{i} \alpha_{j}=\alpha_{j} \alpha_{i} e^{2 \pi i / m}$ and $\alpha_{i}^{m}=1$.

More physically, the zero modes can be understood as a nonzero local density of states, exponentially localized to the domain wall, for a certain class of quasiparticles. This class consists of the fractional excitons $(q / m,-q / m)$, which consists of a charge $q / m$ quasiparticle in the top layer and a charge $-q / m$ quasiparticle in the bottom layer. Consider the operator on the left- or right-moving edge that creates such quasiparticles:

$$
V_{q,-q}^{L}(x, t)=e^{i q\left[\phi_{L 1}(x, t)-\phi_{L 2}(x, t)\right]} .
$$

Next, consider the equal space correlation function of $V_{q,-q}^{L}$ in frequency space:

$$
G_{q,-q}(x, \omega) \equiv\left\langle V_{q,-q}(x, \omega) V_{q,-q}(x,-\omega)\right\rangle .
$$

By analogy with the local electronic density of states, which is given by a similar correlation function for the

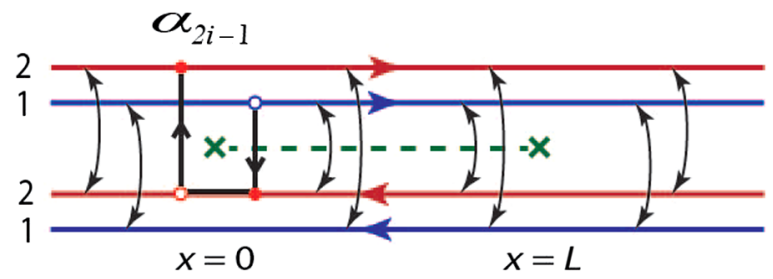

FIG. 3. The defects (genons) can be viewed as domain walls between two different ways of generating an energy gap in the counterpropagating edge states (double arrows indicate interedge electron tunneling). The zero-mode operator $\alpha_{2 i-1}$ corresponds to the quasiparticle hopping process shown in the figure, projected into the ground-state subspace of the edge theory. electron operator, $G_{q,-q}(x, \omega)$ can be interpreted as measuring the "local density of states" of the quasiparticle $(q,-q)$. The parafermion zero mode implies a nonzero density of states at zero energy, exponentially localized to the domain wall:

$$
G_{q,-q}(x, \omega) \sim e^{-|x| / \xi} \delta(\omega),
$$

for $\omega \ll g$, where $g$ sets the energy gap of the domains separated by the domain wall and $\xi \propto 1 / g$ is the correlation length of the gapped edges. The above is valid near the domain wall at $x=0$.

Other ways of creating domain walls between two different ways of generating an energy gap in a single pair of counterpropagating chiral Luttinger liquids have been explored in Refs. [16-18], where similar parafermion zero modes are obtained.

\section{APPENDIX B: EXPONENTIAL LOCALIZATION OF PARAFERMION ZERO MODES IN THE PRESENCE OF GAPLESS MODES}

The parafermion zero modes explained in the previous section are exponentially localized to the domain walls that separate the two different ways of generating an energy gap in the edge states. However, in the experimental setup proposed in the main text, two of the four counterpropagating edge modes may remain gapless while the other two are gapped due to the interlayer backscattering. Here, we show that the exponential localization of the zero modes persists in this situation as well. That is, even in this physical setup,

$G_{q,-q}(x, \omega) \equiv\left\langle V_{q,-q}(x, \omega) V_{q,-q}(x,-\omega)\right\rangle \sim f(x) e^{-|x| / \xi} \delta(\omega)$,

for $\omega \ll E_{g}$, where $E_{g}$ is the energy gap of the gapped part of the edge states, $\xi \propto 1 / E_{g}$, and $f(x)$ is a decaying function of $x$. Equation (B1) holds near a defect at $x=0$.

To understand this, consider the edge theory description of the TLJ in Fig. 2(b). In the main text, we use separate chiral boson fields $\phi_{L(R) 1}, \phi_{L(R) 2}$ to describe the two edges from each layer. Here, for convenience, we describe the whole edge of the first (second) layer by a single chiral boson $\phi_{1(2)}(x)$. Therefore, $\phi_{1,2}(x)$ each live on a circle with perimeter $2 L$ (with $L$ the length of the TLJ). The edge of each layer is a chiral Luttinger liquid, and it is convenient to group the two chiral Luttinger liquids into a nonchiral Luttinger liquid. For this purpose, we define the spatial coordinate $\tilde{x} \in[-L, L]$ of the two edges with opposite chiralities [Fig. 4(c)]. $\tilde{x}$ increases along the counterclockwise direction for layer 1 and the clockwise direction for layer 2. With such a choice, $\phi_{1}$ and $\phi_{2}$ have opposite chirality (defined with respect to $\tilde{x}$ ), and the vertical backscattering induced at the TLJ occurs locally between $\phi_{1}(\tilde{x})$ and $\phi_{2}(\tilde{x})$. The Luttinger liquid Lagrangian can be written as 


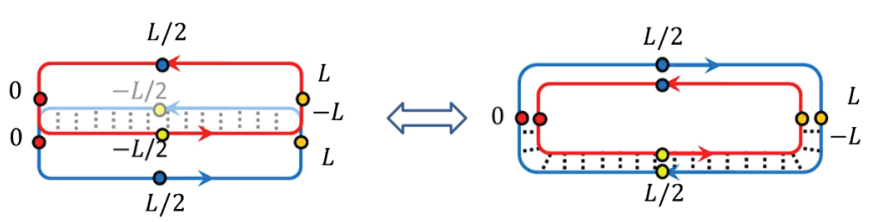

FIG. 4. Edge theory picture of TLJ in Fig. 2(b). The coordinate $\tilde{x}$ increases from $-L$ to $+L$ following the direction indicated by orange, yellow, red, and blue dots. The second layer (blue) is flipped in the image on the right.

$L=\int_{-L}^{L} d \tilde{x}\left[\left(\partial_{t} \varphi\right)^{2}+v\left(\partial_{\tilde{x}} \varphi\right)^{2}+g \theta(-\tilde{x}) \cos (m \varphi)\right]$

with $\varphi=\phi_{1}+\phi_{2}$ and where $\theta(x)$ is a step function. The tunneling induces a gap in the region $\tilde{x} \in[-L, 0]$, and the region $\tilde{x} \in[0, L]$ remains gapless. In this representation, the $m$ topological degenerate ground states are correctly given by the $m$ minima of the $\cos (m \varphi)$ term, with $\varphi$ pinned to the values $2 \pi n / m, n=0,1, \ldots, m-1$. Importantly, in this coordinate the exciton annihilation operator becomes nonlocal: $V_{1,-1}(x)=e^{i \phi_{1}(\tilde{x})-i \phi_{2}(-\tilde{x})}$. Because of the nonlocal commutation relation of chiral boson fields, applying this operator to the ground state causes a domain wall in $\varphi$ in the region $-L<\tilde{x}<0$. Away from the defects at $x=0$ and $x=-L \sim L$, such a domain wall costs an energy of the order of the gap $g$. Therefore, the correlations of this operator must decay exponentially, away from the positions of the defects. At the positions of the defects, this operator keeps the system in the ground-state manifold because it does not create a domain wall in $\varphi$ in the gapped region. Instead, it simply yields a unitary transformation among the ground states by globally flipping the value of $\varphi$ in the gapped region. From this analysis, we see that the zero-bias peak in the exciton local density of states is indeed probing the topologically degenerate states.

\section{APPENDIX C: INTERDIGITATED GATING}

In the experimental setup described in the main text, there are gapless edge states surrounding the TLJs. These edge states do not affect the topological degeneracies, the exponential localization of the zero modes, or the possibility of braiding the genons by tuning the interactions between the parafermion zero modes. Nevertheless, it might be desirable to remove these gapless edge states, while still preserving the topological degeneracy. This can be done simply by alternating the offset in space (see Fig. 5). The tunneling term in the edge states can be modeled as follows:

$$
\delta \mathcal{H}_{\text {tunn }}=\alpha(x) \cos \left(m \tilde{\phi}_{1}\right)+\beta(x) \cos \left(m \tilde{\phi}_{2}\right) .
$$

While $\alpha(x)$ and $\beta(x)$ are never both nonzero simultaneously, at long wavelengths only the average value of $\alpha$ and (a)

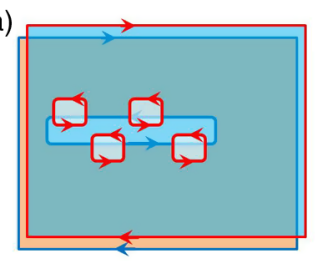

(b)

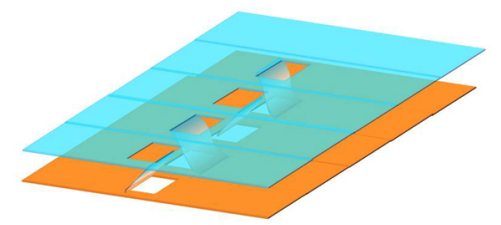

FIG. 5. Alternating the offset of the top and bottom gate will allow us to generate an energy gap for all edge states. At long wavelengths, both twisted tunneling terms, $\sum_{I} \cos \left(m \tilde{\phi}_{I}\right)$ are simultaneously present.

$\beta$ is important. Therefore, both edge states can be gapped in the twisted way on average in this setup.

\section{APPENDIX D: INTERLAYER CURRENT-NOISE CROSS-CORRELATION}

\section{Summary and results}

Here, we present the derivation of the current-noise cross-correlation for the interferometer summarized in the main text. We consider a tunneling Hamiltonian with four QPCs [41,42]:

$$
\begin{aligned}
H_{\text {tunn }}(t) & =\sum_{i=1}^{2}\left[H_{i}(t)+\tilde{H}_{i}(t)\right], \\
H_{i}(t) & =v_{i} \mathcal{O}^{+}\left(x_{i}\right)+v_{i}^{*} \mathcal{O}^{-}\left(x_{i}\right), \\
\tilde{H}_{i}(t) & =\tilde{v}_{i} \tilde{\mathcal{O}}^{+}\left(\tilde{x}_{i}\right)+\tilde{v}_{i}^{*} \tilde{\mathcal{O}}^{-}\left(\tilde{x}_{i}\right),
\end{aligned}
$$

where

$$
v_{i}=\Gamma_{i} e^{-i \omega_{1} t}, \quad \tilde{v}_{i}=\tilde{\Gamma}_{i} e^{-i \omega_{2} t} .
$$

$\Gamma_{i}, \tilde{\Gamma}_{i}$ are the tunneling matrix elements for the different QPCs, and $\omega_{i}=e^{*} V_{i}$, where $V_{i}$ are the potential differences across the QPCs and $e^{*}=e / m$ is the elementary fractional charge of the quasiparticles. We note that in the setup of Fig. 2(c), to lowest order in the tunneling matrix elements, the voltage across each QPC is $V_{i}=V$. For what follows, it is useful to define

$$
x_{12} \equiv x_{1}-x_{2}, \quad \tilde{x}_{12}=\tilde{x}_{1}-\tilde{x}_{2}
$$

The operator $\mathcal{O}^{ \pm}$is the quasiparticle tunneling operator across QPCs enclosing the normal regions of the fluid:

$$
\mathcal{O}^{ \pm}\left(x_{i}\right)=\kappa_{i}^{ \pm} e^{ \pm i\left(\phi_{1 L}+\phi_{1 R}\right)}\left(x_{i}\right),
$$

where $\kappa_{i}$ are Klein factors that ensure that the quasiparticle tunneling operators at different points in space commute with each other, as required. The operators $\tilde{\mathcal{O}}^{ \pm}$are quasiparticle tunneling operators across the twisted line junctions: 


$$
\tilde{\mathcal{O}}^{ \pm}\left(\tilde{x}_{i}\right)=\tilde{\kappa}_{i}^{ \pm} e^{ \pm i\left(\phi_{1 L}+\phi_{2 R}\right)}\left(\tilde{x}_{i}\right),
$$

where $\tilde{\kappa}_{i}^{ \pm}$are also Klein factors. The commutation relations of $\kappa_{i}^{ \pm}$and $\tilde{\kappa}_{i}^{ \pm}$can be determined from the boson commutation relations:

$$
\begin{aligned}
& {\left[\phi_{L I}(x), \phi_{L I}\left(x^{\prime}\right)\right]=i \frac{\pi}{m} \operatorname{sgn}\left(x-x^{\prime}\right),} \\
& {\left[\phi_{R I}(x), \phi_{R I}\left(x^{\prime}\right)\right]=-i \frac{\pi}{m} \operatorname{sgn}\left(x-x^{\prime}\right),}
\end{aligned}
$$

from which we determine that $\left[\kappa_{1}^{r}, \kappa_{2}^{s}\right]=0,\left[\tilde{\kappa}_{1}^{r}, \tilde{\kappa}_{2}^{s}\right]=0$, and

$$
\kappa_{i}^{r} \tilde{\kappa}_{j}^{s}=\tilde{\kappa}_{j}^{s} \kappa_{i}^{r} e^{i \theta_{i j}^{r s}}
$$

where $\theta_{i j}^{r s}=r s \pi \operatorname{sgn}\left(x_{i}-\tilde{x}_{j}\right) / m$.

In the interferometer discussed in the main text, which probes the non-Abelian character of the genons, we require $x_{1}<\tilde{x}_{1}<x_{2}<\tilde{x}_{2}$. However, it is also helpful to compare this with the case $x_{1}<x_{2}<\tilde{x}_{1}<\tilde{x}_{2}$, which does not probe the noncommutativity of the two noncommuting, noncontractible quasiparticle paths.

Now, we let $I_{b 1}$ and $I_{b 2}$ be the current that is backscattered through the normal regions and the TLJs, respectively,

$$
I_{b J}(t)=\frac{d Q_{R J}}{d t}=-i\left[Q_{R J}, H_{\mathrm{tunn}}(t)\right],
$$

where $Q_{R I}=(1 / 2 \pi) \int d x \partial_{x} \phi_{R I}$ is the total charge on the right edge in the Ith layer. Thus,

$$
\begin{aligned}
I_{b 1}(t) & =\frac{i}{m} \sum_{i=1}^{2}\left[\Gamma_{i} e^{-i e^{*} V_{1} t} \mathcal{O}^{+}\left(x_{i}\right)-\text { H.c. }\right] \\
& =\frac{i}{m} \sum_{i=1}^{2}\left[v_{i} \mathcal{O}^{+}\left(x_{i}\right)-v_{i}^{*} \mathcal{O}^{-}\left(x_{i}\right)\right] \\
& =\frac{i}{m} \sum_{i=1}^{2} \sum_{\epsilon_{i}= \pm} \epsilon_{i} v_{i}^{\epsilon_{i}} \mathcal{O}^{\epsilon_{i}}\left(x_{i}\right), \\
I_{b 2}(t) & =\frac{i}{m} \sum_{i=1}^{2}\left[\tilde{\Gamma}_{i} e^{-i e^{*} V_{2} t} \tilde{\mathcal{O}}^{+}\left(\tilde{x}_{i}\right)-\text { H.c. }\right] \\
& =\frac{i}{m} \sum_{i=1}^{2}\left[\tilde{v}_{i} \tilde{\mathcal{O}}^{+}\left(\tilde{x}_{i}\right)-\tilde{v}_{i}^{*} \tilde{\mathcal{O}}^{-}\left(\tilde{x}_{i}\right)\right] \\
& =\frac{i}{m} \sum_{i=1}^{2} \sum_{\epsilon_{i}= \pm} \epsilon_{i} \tilde{v}_{i}^{\epsilon_{i}} \tilde{\mathcal{O}}^{\epsilon_{i}}\left(\tilde{x}_{i}\right) .
\end{aligned}
$$

In the setup of Fig. 2, we find that

$$
\begin{aligned}
& I_{1}=\frac{1}{m} \frac{e^{2}}{h} V+I_{b 1}, \\
& I_{2}=\frac{1}{m} \frac{e^{2}}{h} V+I_{b 2}+I_{g 2},
\end{aligned}
$$

where $I_{1}$ and $I_{2}$ are the currents flowing through the device in the first and second layer [see Fig. 2(c)]. In order to understand $I_{g 2}$, observe that current can be backscattered through the TLJs in two different ways. The first way is through quasiparticle tunneling across the TLJ. The second way is if the outer edge that follows the constriction of the QPC overlaps the gapless edge states that flow around the TLJ. In this case, there can be interlayer electron tunneling between the top and bottom outer edges of the sample, through the edge states surrounding the TLJ. This latter contribution is what we call $I_{g 2}$. Therefore,

$$
\begin{aligned}
S_{12}(t) \equiv & \frac{1}{2}\left\langle I_{1}(t) I_{2}(0)\right\rangle-\left\langle I_{1}(t)\right\rangle\left\langle I_{2}(0)\right\rangle \\
= & \frac{1}{2}\left\langle I_{b 1}(t)\left[I_{b 2}(0)+I_{g 2}(0)\right]\right\rangle \\
& -\left\langle I_{1}(t)\right\rangle\left\langle\left[I_{2}(0)+I_{g 2}(0)\right]\right\rangle .
\end{aligned}
$$

We are interested in the limit $|t| \gg 1 / T$, in which case it will become clear that $I_{g 2}$, which involves only electron tunneling, will not have long-time cross-correlations between the quasiparticle tunneling current $I_{b 1}$.

Therefore, we compute the following interlayer noise cross-correlation:

$S_{12}(t)=\frac{1}{2}\left\langle\left\{I_{b 1}(t), I_{b 2}(0)\right\}\right\rangle-\left\langle I_{b 1}(t)\right\rangle\left\langle I_{b 2}(0)\right\rangle$.

Note that the expectation values are given by

$$
\langle A(t)\rangle=\left\langle T_{C} A(t) e^{-i \int_{C} H_{\mathrm{tunn}}(\tau) d \tau}\right\rangle_{0}
$$

where the $\langle\cdot\rangle_{0}$ indicates an expectation value in the unperturbed theory. $C$ indicates the Keldysh contour, and $T_{C}$ is time ordering in the Keldsyh contour. In our calculation, we drop the subscript and assume all expectation values are in the unperturbed theory.

We first present the result of the calculation. We find that to lowest oder in the QPC tunneling matrix elements,

$$
\begin{aligned}
S_{12}(|t| \gg 1 / T) \\
=\frac{(\pi T)^{4 / m-2}}{m^{2}}\left\{\left|\Gamma_{1}\right|^{2}\left|\tilde{\Gamma}_{1} \tilde{\Gamma}_{2}\right| \theta(-t) S_{12}^{(1)}\left(\bar{\omega}_{2}, \bar{\omega}_{1}, \overline{\tilde{x}}_{12}, \tilde{\varphi}_{12}\right)\right. \\
\quad+\left|\tilde{\Gamma}_{1}\right|^{2}\left|\Gamma_{1} \Gamma_{2}\right| \theta(t) S_{12}^{(1)}\left(\bar{\omega}_{1}, \bar{\omega}_{2}, \bar{x}_{12}, \varphi_{12}\right) \\
\quad+\left|\Gamma_{1} \Gamma_{2} \tilde{\Gamma}_{1} \tilde{\Gamma}_{2}\right|\left[S_{12}^{(2)}\left(\bar{\omega}_{1}, \bar{\omega}_{2}, \bar{x}_{12}, \overline{\tilde{x}}_{12}, \tilde{\varphi}_{12}, \varphi_{12}\right) \theta(t)\right. \\
\left.\left.\quad+S_{12}^{(2)}\left(\bar{\omega}_{2}, \bar{\omega}_{1}, \overline{\tilde{x}}_{12}, \bar{x}_{12}, \varphi_{12}, \tilde{\varphi}_{12}\right) \theta(-t)\right]\right\},
\end{aligned}
$$

where we have defined the dimensionless quantities 


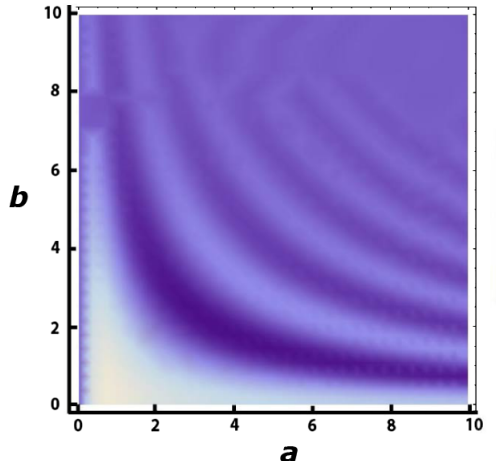

(a)
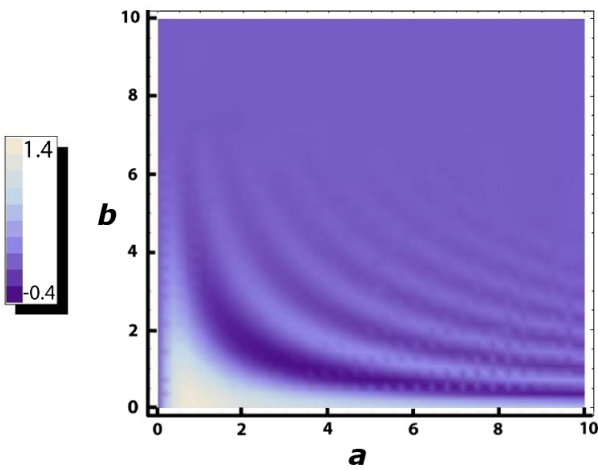

(b)

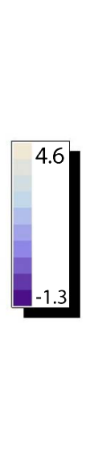

FIG. 6. Density plots of (a) $-c_{r}(a, b) / 2 \sin (\pi / m)$, (b) $f_{-}(a, b)$, and (c) $f_{+}(a, b)$, for $m=3$.

$$
\begin{aligned}
\bar{\omega}_{1} \equiv \frac{\hbar}{\pi k_{B}} \frac{\omega_{1}}{T}, & \bar{\omega}_{2} \equiv \frac{\hbar}{\pi k_{B}} \frac{\omega_{2}}{T}, \\
\bar{x}_{12} \equiv \frac{\pi k_{B}}{\hbar v} T x_{12}, & \overline{\tilde{x}}_{12} \equiv \frac{\pi k_{B}}{\hbar v} T \tilde{x}_{12}
\end{aligned}
$$

and $\varphi_{12}$ and $\tilde{\varphi}_{12}$ are the phase differences between the tunneling matrix elements of the QPCs: $\Gamma_{1} \Gamma_{2}^{*}=\left|\Gamma_{1} \Gamma_{2}\right| e^{i \varphi_{12}}$ and $\tilde{\Gamma}_{1} \tilde{\Gamma}_{2}^{*}=\left|\tilde{\Gamma}_{1} \tilde{\Gamma}_{2}\right| e^{i \tilde{\varphi}_{12}}$. Note that $S_{12}^{(i)}$ depend on only dimensionless quantities.

$$
\begin{aligned}
& S_{12}^{(1)}\left(\bar{\omega}_{2}, \bar{\omega}_{1}, x, \tilde{\varphi}_{12}\right) \\
& \quad=4 c_{r}\left(\bar{\omega}_{2}, 2 x\right)\left\{[\cos (2 \pi / m)-1] \cos \tilde{\varphi}_{12} f_{-}\left(\bar{\omega}_{1}, 0\right)\right. \\
& \left.\quad-\sin \tilde{\varphi}_{12} \sin (2 \pi / m) f_{+}\left(\bar{\omega}_{1}, 0\right)\right\}, \\
& S_{12}^{(2)}\left(\bar{\omega}_{1}, \bar{\omega}_{2}, x, y, \tilde{\varphi}_{12}, \varphi_{12}\right) \\
& \quad=8 c_{r}\left(\bar{\omega}_{1}, 2 x\right) \cos \tilde{\varphi}_{12}\left\{2[\cos (\pi / m)-1] \cos \left(\varphi_{12}\right) c_{r}\left(\bar{\omega}_{2}, 2 y\right)\right. \\
& \left.\quad-\sin (\pi / m) \sin \left(\varphi_{12}\right) f_{+}\left(\bar{\omega}_{2}, y\right)\right\} .
\end{aligned}
$$

The functions $f_{ \pm}(\bar{\omega}, \bar{x})$ are defined as

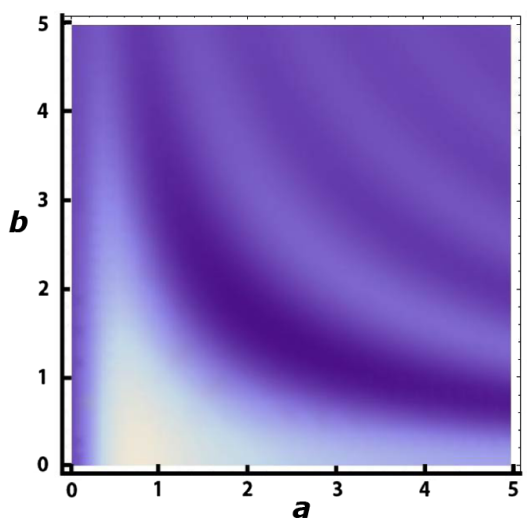

(a)

$$
\begin{aligned}
f_{ \pm}(\bar{\omega}, \bar{x}) \equiv & \int_{-\infty}^{\infty} e^{i \bar{\omega} t} d t\left[e^{-(i \pi / 2 m)[\operatorname{sgn}(t+\bar{x})+\operatorname{sgn}(t-\bar{x})]}\right. \\
& \left. \pm e^{(i \pi / 2 m)[\operatorname{sgn}(t+\bar{x})+\operatorname{sgn}(t-\bar{x})]}\right] \\
& \times\left(\frac{1}{\sinh |t+\bar{x}| \sinh |t-\bar{x}|}\right)^{1 / m}
\end{aligned}
$$

while $c_{r}(a, b)$ is defined as

$$
\begin{aligned}
c_{r}(a, b) \equiv & -2 \sin (\pi / m) \\
& \times \operatorname{Im}\left\{e^{i a b / 2} \int_{0}^{\infty} d t e^{i a t}\left(\frac{1}{\sinh [t] \sinh [t+|b|]}\right)^{1 / m}\right\} .
\end{aligned}
$$

As a check, observe that when $m=1$ and $m \rightarrow \infty$, the two noncontractible paths for quasiparticle propagation commute, and, therefore, this noise cross-correlation should vanish at long times, which it indeed does.

In Fig. 6, we plot the functions $f_{ \pm}(a, b)$ and $-c_{r}(a, b) / \sin (\pi / m)$. In Fig. 7, we plot $S_{12}^{(i)}(a, b, a, b, 0,0)$,

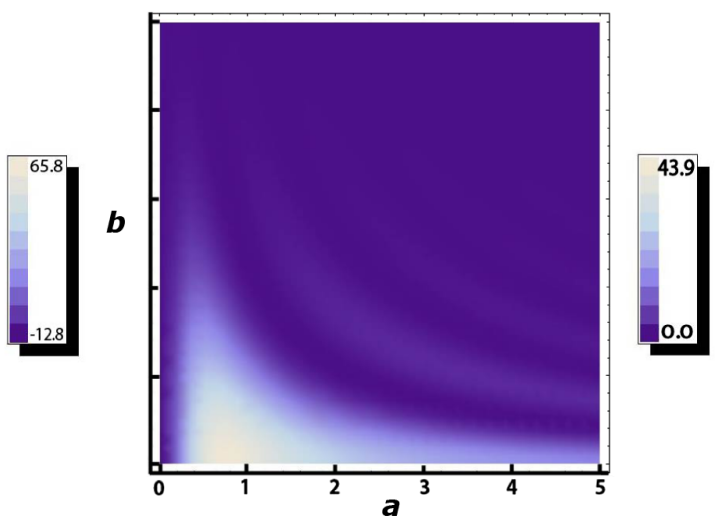

(b)

FIG. 7. Density plots of (a) $S_{12}^{(1)}(a, b, a, b, 0,0)$ and (b) $-S_{12}^{(2)}(a, b, a, b, 0,0)$, for $m=3$. Both are zero when $a=b=0$ reaches a maximum for $a$ and $b$ of order 1 , and decay to zero with increasing $a$ and $b$. 
for $i=1,2$, that is, for the case where the voltage differences between all QPCs is the same, $x_{12}=\tilde{x}_{12}$, and for zero-phase differences $\varphi_{12}$ and $\tilde{\varphi}_{12}$. We see that when $a$ and $b$ are of order $1, S_{12}^{(i)}$ reach their maximum value and decay quickly as $a$ and $b$ increase. From this, we see that the noise cross-correlation will require the dimensionless parameters $\bar{\omega}_{i}, \bar{x}_{12}$, and $\tilde{\bar{x}}_{12}$ to all be of order 1 . If we assume $T \approx 50-100 \mathrm{mK}$ and $v \approx 10^{5} \mathrm{~m} / \mathrm{s}$, we see that this requires $x_{12}$ and $\tilde{x}_{12}$ be on the order of several $\mu \mathrm{m}$, which is reasonable for realistic device dimensions.

\section{Klein factors}

Here, we determine the Keldysh-contour ordered correlation functions of the Klein factors, which are required for the calculation. To do this, first, we observe that the twopoint function for Klein factors that commute with each other should be one. Next, we observe that the correlation functions of several Klein factors should factor into twopoint functions. In order to obtain the two-point function of noncommuting Klein factors, it is helpful to represent the Klein factors in terms of bosonic operators [43]:

$$
\kappa_{i}^{ \pm}=: e^{ \pm i \sqrt{1 / m} \theta_{i}}:, \quad \tilde{\kappa}_{i}^{ \pm}=: e^{ \pm i \sqrt{1 / m} \tilde{\theta}_{i}}:
$$

where $\theta_{i}, \tilde{\theta}_{i}$ are bosonic operators, and for what follows, we leave the normal ordering $: \cdots:$ implicit. Using the fact that $: e^{A}:: e^{B}:=: e^{B}:: e^{A}: e^{[A, B]}$ for cases where $[A, B]$ is a $c$ number, we find that the commutation relation of the Klein factors is reproduced when

$\left[\theta_{i}, \tilde{\theta}_{j}\right]=-i \pi \operatorname{sgn}\left(x_{i}-\tilde{x}_{j}\right), \quad\left[\theta_{i}, \theta_{j}\right]=\left[\tilde{\theta}_{i}, \tilde{\theta}_{j}\right]=0$.

Since $\theta_{i}$ and $\tilde{\theta}_{j}$ are conjugates, we can form a harmonic oscillator algebra:

$a_{i j}=\frac{1}{\sqrt{2 \pi}}\left(\theta_{i}+i \tilde{\theta}_{j}\right), \quad a_{i j}^{\dagger}=\frac{1}{\sqrt{2 \pi}}\left(\theta_{i}-i \tilde{\theta}_{j}\right)$,

such that

$\left[a_{i j}, a_{i j}^{\dagger}\right]=-\operatorname{sgn}\left(x_{i}-\tilde{x}_{j}\right), \quad\left\langle a_{i j} a_{i j}^{\dagger}\right\rangle=\frac{1-\operatorname{sgn}\left(x_{i}-\tilde{x}_{j}\right)}{2}$.

We suppose that the Hamiltonian for these bosonic fields is zero, as they have no dynamics. From this, we conclude that

$$
\begin{aligned}
& \left\langle\theta_{i}(t) \theta_{i}(0)\right\rangle=\left\langle\tilde{\theta}_{i}(t) \tilde{\theta}_{i}(0)\right\rangle=\pi / 2, \\
& \left\langle\theta_{i}(t) \tilde{\theta}_{j}(0)\right\rangle=-\left\langle\tilde{\theta}_{j}(t) \theta_{i}(0)\right\rangle=-\frac{i \pi}{2} \operatorname{sgn}\left(x_{i}-\tilde{x}_{j}\right) .
\end{aligned}
$$

From this, we conclude that the Keldysh contour ordered correlation function is $g_{i j}^{\eta_{1} \eta_{2}}(t) \equiv\left\langle T_{C} \theta_{i}\left(t^{\eta_{1}}\right) \tilde{\theta}_{j}\left(0^{\eta_{2}}\right)\right\rangle=-i \frac{\pi}{2} \operatorname{sgn}\left(x_{i}-\tilde{x}_{j}\right) \chi_{\eta_{1} \eta_{2}}(t)$,

where

$$
\chi_{\eta_{1} \eta_{2}}(t)=\frac{\eta_{1}+\eta_{2}}{2} \operatorname{sgn}(t)-\frac{\eta_{1}-\eta_{2}}{2} .
$$

Therefore,

$$
\left\langle T_{C} \kappa_{i}^{\epsilon}\left(t^{\eta_{1}}\right) \tilde{\kappa}_{j}^{\tilde{\epsilon}}\left(0^{\eta_{2}}\right)\right\rangle=e^{i \epsilon \tilde{\epsilon}(\pi / 2 m) \chi_{\eta_{1} \eta_{2}}(t) \operatorname{sgn}\left(x_{i}-\tilde{x}_{j}\right)} .
$$

For later reference, we need the following correlation function:

$$
\begin{aligned}
& \left\langle T_{C} \kappa_{\alpha_{1}}^{\epsilon_{1}}\left(t^{\eta}\right) \kappa_{\beta_{1}}^{-\epsilon_{1}}\left(t^{\eta_{1}}\right) \tilde{\kappa}_{\alpha_{2}}^{\epsilon_{2}}\left(0^{-\eta}\right) \tilde{\kappa}_{\beta_{2}}^{-\epsilon_{2}}\left(0^{\eta_{2}}\right)\right\rangle \\
& =\left\langle T_{C} e^{i \sqrt{1 / m} \epsilon_{1} \theta_{\alpha_{1}}\left(t^{\eta}\right)} e^{-i \sqrt{1 / m} \epsilon_{1} \theta_{\beta_{1}}\left(t^{\eta_{1}}\right)}\right. \\
& \left.\quad \times e^{i \sqrt{1 / m} \epsilon_{2} \tilde{\theta}_{\alpha_{2}}\left(0^{-\eta}\right)} e^{-\epsilon_{2} i \sqrt{1 / m} \tilde{\theta}_{\beta_{2}}\left(0^{\eta_{2}}\right)}\right\rangle .
\end{aligned}
$$

To calculate this, we use the fact that $\left\langle T_{C} e^{A_{1}} \cdots e^{A_{n}}\right\rangle=$ $e^{\sum_{i<j}^{n}\left\langle T_{C} A_{i} A_{j}\right\rangle}$, where in the Klein factor case, we include only correlation functions between noncommuting variables. Therefore,

$$
\begin{aligned}
& \left\langle T_{C} \kappa_{\alpha_{1}}^{\epsilon_{1}}\left(t^{\eta}\right) \kappa_{\beta_{1}}^{-\epsilon_{1}}\left(t^{\eta_{1}}\right) \tilde{\kappa}_{\alpha_{2}}^{\epsilon_{2}}\left(0^{-\eta}\right) \tilde{\kappa}_{\beta_{2}}^{-\epsilon_{2}}\left(0^{\eta_{2}}\right)\right\rangle \\
& =e^{i(\pi / 2 m) \epsilon_{1} \epsilon_{2}\left[\chi_{\eta-\eta}(t) s_{\alpha_{1} \alpha_{2}}-\chi_{\eta_{\eta_{2}}}(t) s_{\alpha_{1} \beta_{2}}-\chi_{\eta_{1}-\eta}(t) s_{\beta_{1} \alpha_{2}}+\chi_{\eta_{1} \eta_{2}}(t) s_{\beta_{1} \beta_{2}}\right]} \\
& = \begin{cases}e^{-i\left(\epsilon_{1} \epsilon_{2} / 2 m\right) \pi\left[\eta\left(s_{\alpha_{1} \alpha_{2}}-s_{\beta_{1} \alpha_{2}}\right)+\eta_{2}\left(s_{\alpha_{1} \beta_{2}}-s_{\beta_{1} \beta_{2}}\right)\right]} & t>0 \\
e^{i\left(\epsilon_{1} \epsilon_{2} / 2 m\right) \pi\left[\eta\left(s_{\alpha_{1} \beta_{2}}-s_{\alpha_{1} \alpha_{2}}\right)+\eta_{1}\left(s_{\beta_{1} \alpha_{2}}-s_{\beta_{1} \beta_{2}}\right)\right]} & t<0,\end{cases}
\end{aligned}
$$

where $s_{\alpha \beta} \equiv \operatorname{sgn}\left(x_{\alpha}-\tilde{x}_{\beta}\right)$. When $x_{1}<x_{2}<\tilde{x}_{1}<\tilde{x}_{2}$, $s_{\alpha \beta}=-1$ for all $\alpha, \beta$, and, therefore,

$$
\left\langle T_{C} \kappa_{\alpha_{1}}^{\epsilon_{1}}\left(t^{\eta}\right) \kappa_{\beta_{1}}^{-\epsilon_{1}}\left(t^{\eta_{1}}\right) \tilde{\kappa}_{\alpha_{2}}^{\epsilon_{2}}\left(0^{-\eta}\right) \tilde{\kappa}_{\beta_{2}}^{-\epsilon_{2}}\left(0^{\eta_{2}}\right)\right\rangle=1 .
$$

When $x_{1}<\tilde{x}_{1}<x_{2}<\tilde{x}_{2}$, which is the case of interest for our proposed interferometer, we instead have

$$
\begin{aligned}
& \left\langle T_{C} \kappa_{\alpha_{1}}^{\epsilon_{1}}\left(t^{\eta}\right) \kappa_{\beta_{1}}^{-\epsilon_{1}}\left(t^{\eta_{1}}\right) \tilde{\kappa}_{\alpha_{2}}^{\epsilon_{2}}\left(0^{-\eta}\right) \tilde{\kappa}_{\beta_{2}}^{-\epsilon_{2}}\left(0^{\eta_{2}}\right)\right\rangle \\
& = \begin{cases}e^{-i\left(\epsilon_{1} \epsilon_{2} / m\right)\left(\alpha_{1}-\beta_{1}\right) \pi\left[\eta \delta_{\alpha_{2} 1}+\eta_{2} \delta_{\beta_{2}}\right]} & \text { if } t>0 \\
e^{-i\left(\epsilon_{1} \epsilon_{2} / m\right)\left(\alpha_{2}-\beta_{2}\right) \pi\left[-\eta \delta_{\alpha_{1} 2}+\eta_{1} \delta_{\beta_{1}}\right]} & \text { if } t<0 .\end{cases}
\end{aligned}
$$

\section{Boson correlation functions}

We need the following correlation functions:

$$
\begin{aligned}
G_{\eta \eta^{\prime}}\left(x-x^{\prime}, t-t^{\prime}\right) & \equiv\left\langle T_{C} e^{i \epsilon \phi\left(x, t^{\prime \prime}\right)} e^{-i \epsilon \phi\left(x^{\prime}, t^{\prime \eta^{\prime}}\right)}\right\rangle \\
& =e^{\left\langle T_{C} \phi\left(x, t^{\prime}\right) \phi\left(x^{\prime}, t^{\prime \eta^{\prime}}\right)\right\rangle} .
\end{aligned}
$$


At finite temperatures, this is (setting the velocities of the left and right movers to unity)

$$
G_{\eta_{1} \eta_{2}}(x, t)=\left(\frac{(\pi T)^{2}}{\sin \left\{\pi T\left[\delta+i \chi_{\eta_{1} \eta_{2}}(t)(t+x)\right]\right\} \sin \left\{\pi T\left[\delta+i \chi_{\eta_{1} \eta_{2}}(t)(t-x)\right]\right\}}\right)^{1 / m},
$$

where

$$
\chi_{\eta_{1} \eta_{2}}(t)=\frac{\left(\eta_{1}+\eta_{2}\right)}{2} \operatorname{sgn}(t)-\frac{\left(\eta_{1}-\eta_{2}\right)}{2} .
$$

Taking the limit $\delta \rightarrow 0^{+}$, we can replace the above by

$$
\begin{aligned}
G_{\eta_{1} \eta_{2}}(x, t)= & e^{-i(\pi / 2 m) \chi_{\eta_{1} \eta_{2}}(t)[\operatorname{sgn}(t+x)+\operatorname{sgn}(t-x)]} \\
& \times\left(\frac{(\pi T)^{2}}{\sinh [\pi T|t+x|] \sinh [\pi T|t-x|]}\right)^{1 / m} .
\end{aligned}
$$

$$
\begin{aligned}
G_{\eta_{1} \eta_{2}}^{L / R}(x, t)= & \left\langle T_{C} e^{i \epsilon \phi_{L / R}\left(x, t^{\eta_{1}}\right)} e^{-i \epsilon \phi_{L / R}\left(0,0^{\eta_{2}}\right)}\right\rangle \\
= & e^{-i(\pi / 2 m) \chi_{\eta_{1} \eta_{2}}(t) \operatorname{sgn}(|v| t \pm x)} \\
& \times\left(\frac{(\pi T)}{\sinh \left[\pi T|| v_{L / R}|t \pm x|\right]}\right)^{1 / m} .
\end{aligned}
$$

The chiral correlator, involving only the left- or rightmoving modes (and reinserting the velocities $v_{L / R}$ for the left or right movers), is

\section{Details of calculation}

Here, we describe in detail the calculation of $S_{12}(t)$, to lowest order in the tunneling matrix elements. We have [recall Eqs. (D1) and (D13)]

$$
\begin{aligned}
\frac{1}{2}\left\langle\left\{I_{b 1}(t), I_{b 2}(0)\right\}\right\rangle & =\frac{1}{4} \sum_{\eta= \pm}(-i)^{2} \int_{C} d \tau_{1} d \tau_{2}\left\langle T_{C} I_{b 1}\left(t^{\eta}\right) H_{\text {tunn }}\left(\tau_{1}\right) I_{b 2}\left(0^{-\eta}\right) H_{\text {tunn }}\left(\tau_{2}\right)\right\rangle \\
& =-\frac{1}{2} \sum_{\eta, \eta_{1}, \eta_{2}= \pm} \int_{-\infty}^{\infty} \eta_{1} \eta_{2} d \tau_{1}^{\eta_{1}} d \tau_{2}^{\eta_{2}}\left\langle T_{C} I_{b 1}\left(t^{\eta}\right) H\left(\tau_{1}^{\eta_{1}}\right) I_{b 2}\left(0^{-\eta}\right) \tilde{H}\left(\tau_{2}^{\eta_{2}}\right)\right\rangle
\end{aligned}
$$

Consider the correlation function:

$$
\begin{aligned}
\left\langle T_{C} I_{b 1}\left(t^{\eta}\right) H\left(\tau_{1}^{\eta_{1}}\right) I_{b 2}\left(0^{-\eta}\right) \tilde{H}\left(\tau_{2}^{\eta_{2}}\right)\right\rangle= & \frac{i^{2}}{m^{2}} \sum_{\alpha_{1}, \alpha_{2}, \beta_{1}, \beta_{2}} \sum_{\epsilon_{\alpha_{1}}} \sum_{\beta_{1} \epsilon_{\alpha_{2}}} \epsilon_{\beta_{2}} v_{\alpha_{1}}^{\epsilon_{\alpha_{1}}} v_{\beta_{1}}^{\epsilon_{\beta_{1}}} v_{\alpha_{2}}^{\epsilon_{\alpha_{2}}} v_{\beta_{2}}^{\epsilon_{\beta_{2}}} \epsilon_{\alpha_{1}} \epsilon_{\alpha_{2}} \\
& \times\left\langle T_{C} \mathcal{O}^{\epsilon_{\alpha_{1}}}\left(x_{\alpha_{1}}, t^{\eta}\right) \mathcal{O}^{\epsilon_{\beta_{1}}}\left(x_{\beta_{1}}, \tau_{1}^{\eta_{1}}\right) \tilde{\mathcal{O}}^{\epsilon_{\alpha_{2}}}\left(\tilde{x}_{\alpha_{2}}, 0^{-\eta}\right) \tilde{\mathcal{O}}^{\epsilon_{\beta_{2}}}\left(\tilde{x}_{\beta_{2}}, \tau_{2}^{\eta_{2}}\right)\right\rangle .
\end{aligned}
$$

The above correlation function vanishes unless $\epsilon_{\alpha_{1}}=-\epsilon_{\beta_{1}}$ and $\epsilon_{\alpha_{2}}=-\epsilon_{\beta_{2}}$. Therefore,

$$
\begin{aligned}
\left\langle T_{C} I_{b 1}\left(t^{\eta}\right) H\left(\tau_{1}^{\eta_{1}}\right) I_{b 2}\left(0^{-\eta}\right) \tilde{H}\left(\tau_{2}^{\eta_{2}}\right)\right\rangle= & \frac{1}{m^{2}} \sum_{\alpha_{1}, \alpha_{2}, \beta_{1}, \beta_{2}} \sum_{\epsilon_{1} \epsilon_{2}} v_{\alpha_{1}}^{\epsilon_{1}} v_{\beta_{1}}^{-\epsilon_{1}} v_{\alpha_{2}}^{\epsilon_{2}} v_{\beta_{2}}^{-\epsilon_{2}} \epsilon_{1} \epsilon_{2}\left\langle T_{C} \kappa_{\alpha_{1}}^{\epsilon_{1}}\left(t^{\eta}\right) \kappa_{\beta_{1}}^{-\epsilon_{1}}\left(\tau_{1}^{\eta_{1}}\right) \tilde{\kappa}_{\alpha_{2}}^{\epsilon_{2}}\left(0^{-\eta}\right) \tilde{\kappa}_{\beta_{2}}^{-\epsilon_{2}}\left(\tau_{2}^{\eta_{2}}\right)\right\rangle \\
& \times\left\langle T_{C} e^{i \varepsilon_{1} \phi_{1 R}\left(x_{\alpha_{1}}, t^{\eta \eta}\right)} e^{-i \varepsilon_{1} \phi_{1 R}\left(x_{\beta_{1}}, \tau_{1}^{\eta_{1}}\right)}\right\rangle\left\langle T_{C} e^{i \varepsilon_{2} \phi_{2 R}\left(\tilde{x}_{\alpha_{2}}, 0^{-\eta}\right)} e^{-i \varepsilon_{2} \phi_{2 R}\left(\tilde{x}_{\beta_{2}}, \tau_{2}^{\eta_{2}}\right)}\right\rangle \\
& \times\left\langle T_{C} e^{i \epsilon_{1} \phi_{1 L}\left(x_{\alpha_{1}}, t^{\eta}\right)} e^{-i \epsilon_{1} \phi_{1 L}\left(x_{\beta_{1}}, \tau_{1}^{\eta_{1}}\right)} e^{i \epsilon_{2} \phi_{1 L}\left(x_{\alpha_{2}}, 0^{\eta \eta}\right)} e^{-i \epsilon_{2} \phi_{1 L}\left(\beta_{2}, \tau_{2}^{\eta_{2}}\right)}\right\rangle .
\end{aligned}
$$

Recall that in our notation, the superscript - implies complex conjugation, not inverse.

$$
\begin{aligned}
\left\langle T_{C} I_{b 1}\left(t^{\eta}\right) H\left(\tau_{1}^{\eta_{1}}\right) I_{b 2}\left(0^{-\eta}\right) \tilde{H}\left(\tau_{2}^{\eta_{2}}\right)\right\rangle=- & \frac{1}{m^{2}} \sum_{\epsilon_{1}, \epsilon_{2}= \pm \alpha_{1}, \beta_{1}, \alpha_{2}, \beta_{2}} \epsilon_{1} \epsilon_{2} \Gamma_{\alpha_{1}}^{\epsilon_{1}} \Gamma_{\beta_{1}}^{-\epsilon_{1}} \Gamma_{\alpha_{2}}^{\epsilon_{2}} \Gamma_{\beta_{2}}^{-\epsilon_{2}} e^{i \epsilon_{1} \omega_{1}\left(t-\tau_{1}\right)+i \epsilon_{2} \omega_{2}\left(-\tau_{2}\right)} \\
& \times\left\langle T_{C} \kappa_{\alpha_{1}}^{\epsilon_{1}}\left(t^{\eta}\right) \kappa_{\beta_{1}}^{-\epsilon_{1}}\left(\tau_{1}^{\eta_{1}}\right) \tilde{\kappa}_{\alpha_{2}}^{\epsilon_{2}}\left(0^{-\eta}\right) \tilde{\kappa}_{\beta_{2}}^{-\epsilon_{2}}\left(\tau_{2}^{\eta_{2}}\right)\right\rangle \\
& \times G_{-\eta \eta_{2}}^{R, v_{1}}\left(\tilde{x}_{\alpha_{2}}-\tilde{x}_{\beta_{2}},-\tau_{2}\right) G_{\eta \eta_{1}}^{R, v_{2}}\left(x_{\alpha_{1}}-x_{\beta_{1}}, t-\tau_{1}\right) \\
& \times\left\langle T_{C} e^{i \epsilon_{1} \phi_{L 1}\left(x_{\alpha_{1}}, t^{\eta}\right)} e^{-i \epsilon_{1} \phi_{L 1}\left(x_{\beta_{1}}, \tau_{1}^{\eta_{1}}\right)} e^{i \epsilon_{2} \phi_{L 1}\left(\tilde{x}_{\alpha_{2}}, 0^{-\eta}\right)} e^{-i \epsilon_{2} \phi_{L 1}\left(\tilde{x}_{\beta_{2}}, \tau_{2}^{\eta_{2}}\right)}\right\rangle .
\end{aligned}
$$


This gives

$$
\begin{aligned}
& \left\langle T_{C} I_{b 1}\left(t^{\eta}\right) H\left(\tau_{1}^{\eta_{1}}\right) I_{b 2}\left(0^{-\eta}\right) \tilde{H}\left(\tau_{2}^{\eta_{2}}\right)\right\rangle=-\frac{1}{m^{2}} \sum_{\epsilon_{1}, \epsilon_{2}= \pm \alpha_{1}, \beta_{1}, \alpha_{2}, \beta_{2}} \epsilon_{1} \epsilon_{2} \Gamma_{\alpha_{1}}^{\epsilon_{1}} \Gamma_{\beta_{1}}^{-\epsilon_{1}} \Gamma_{\alpha_{2}}^{\epsilon_{2}} \Gamma_{\beta_{2}}^{-\epsilon_{2}} e^{i \epsilon_{1} \omega_{1}\left(t-\tau_{1}\right)+i \epsilon_{2} \omega_{2}\left(-\tau_{2}\right)} \\
& \times\left\langle T_{C} \kappa_{\alpha_{1}}^{\epsilon_{1}}\left(t^{\eta}\right) \kappa_{\beta_{1}}^{-\epsilon_{1}}\left(\tau_{1}^{\eta_{1}}\right) \tilde{\kappa}_{\alpha_{2}}^{\epsilon_{2}}\left(0^{-\eta}\right) \tilde{\kappa}_{\beta_{2}}^{-\epsilon_{2}}\left(\tau_{2}^{\eta_{2}}\right)\right\rangle \\
& \times G_{-\eta \eta_{2}}^{R, v_{2}}\left(\tilde{x}_{\alpha_{2}}-\tilde{x}_{\beta_{2}},-\tau_{2}\right) G_{-\eta \eta_{2}}^{L, v_{1 L}}\left(\tilde{x}_{\alpha_{2}}-\tilde{x}_{\beta_{2}},-\tau_{2}\right) \\
& \times G_{\eta \eta_{1}}^{R ; v_{1 R}}\left(x_{\alpha_{1}}-x_{\beta_{1}}, t-\tau_{1}\right) G_{\eta \eta_{1}}^{L ; v_{1 L}}\left(x_{\alpha_{1}}-x_{\beta_{1}}, t-\tau_{1}\right) \\
& \times\left(\frac{G_{\eta \eta_{2}}^{L, v_{1 L}}\left(x_{\alpha_{1}}-\tilde{x}_{\beta_{2}}, t-\tau_{2}\right) G_{\eta_{1}-\eta}^{L, v_{1 L}}\left(x_{\beta_{1}}-\tilde{x}_{\alpha_{2}}, \tau_{1}\right)}{G_{\eta-\eta}^{L, v_{1 L}}\left(x_{\alpha_{1}}-\tilde{x}_{\alpha_{2}}, t\right) G_{\eta_{1} \eta_{2}}^{L, v_{1 L}}\left(x_{\beta_{1}}-\tilde{x}_{\beta_{2}}, \tau_{1}-\tau_{2}\right)}\right)^{\epsilon_{1} \epsilon_{2}} .
\end{aligned}
$$

The Green functions $G_{\eta \eta^{\prime}}^{L / R}(x, t)$ decay exponentially in $t$; therefore, the integral will be dominated only by regions where $\tau_{1} \approx t$ and $\tau_{2} \approx 0$. Furthermore, we are interested in the long-time behavior, where $t$ is large. In this limit, the ratio in parentheses above will go to one:

$$
\left(\frac{G_{\eta \eta_{2}}^{L, v_{1 L}}\left(x_{\alpha_{1}}-\tilde{x}_{\beta_{2}}, t-\tau_{2}\right) G_{\eta_{1}-\eta}^{L, v_{1 L}}\left(x_{\beta_{1}}-\tilde{x}_{\alpha_{2}}, \tau_{1}\right)}{G_{\eta-\eta}^{L, v_{1 L}}\left(x_{\alpha_{1}}-\tilde{x}_{\alpha_{2}}, t\right) G_{\eta_{1} \eta_{2}}^{L, v_{1 L}}\left(x_{\beta_{1}}-\tilde{x}_{\beta_{2}}, \tau_{1}-\tau_{2}\right)}\right) \rightarrow 1,
$$

when $t \approx \tau_{1} \rightarrow \infty$ and $|t| \gg\left|\tau_{2}\right|, t \gg\left|x_{\alpha}-\tilde{x}_{\beta}\right|$. Therefore,

$$
\begin{aligned}
\left\langle T_{C} I_{b 1}\left(t^{\eta}\right) H\left(\tau_{1}^{\eta_{1}}\right) I_{b 2}\left(0^{-\eta}\right) \tilde{H}\left(\tau_{2}^{\eta_{2}}\right)\right\rangle \approx & -\frac{1}{m^{2}} \sum_{\epsilon_{1}, \epsilon_{2}= \pm \alpha_{1}, \beta_{1}, \alpha_{2}, \beta_{2}} \epsilon_{1} \epsilon_{2} \Gamma_{\alpha_{1}}^{\epsilon_{1}} \Gamma_{\beta_{1}}^{-\epsilon_{1}} \Gamma_{\alpha_{2}}^{\epsilon_{2}} \Gamma_{\beta_{2}}^{-\epsilon_{2}} e^{i \epsilon_{1} \omega_{1}\left(t-\tau_{1}\right)+i \epsilon_{2} \omega_{2}\left(-\tau_{2}\right)} \\
& \times\left\langle T_{C} \kappa_{\alpha_{1}}^{\epsilon_{1}}\left(t^{\eta}\right) \kappa_{\beta_{1}}^{-\epsilon_{1}}\left(\tau_{1}^{\eta_{1}}\right) \tilde{\kappa}_{\alpha_{2}}^{\epsilon_{2}}\left(0^{-\eta}\right) \tilde{\kappa}_{\beta_{2}}^{-\epsilon_{2}}\left(\tau_{2}^{\eta_{2}}\right)\right\rangle \\
& \times G_{-\eta \eta_{2}}^{v_{2 R} ; v_{1 L}}\left(\tilde{x}_{\alpha_{2}}-\tilde{x}_{\beta_{2}},-\tau_{2}\right) G_{\eta \eta_{1}}^{v_{1 R} ; v_{1 L}}\left(x_{\alpha_{1}}-x_{\beta_{1}}, t-\tau_{1}\right) .
\end{aligned}
$$

Therefore, so far we have, for large $t$,

$$
\begin{aligned}
S_{12}(t)= & \frac{1}{2} \frac{1}{m^{2}} \sum_{\eta, \eta_{1}, \eta_{2} \epsilon_{1}, \epsilon_{2}= \pm \alpha_{1}, \beta_{1}, \alpha_{2}, \beta_{2}} \epsilon_{1} \epsilon_{2} \Gamma_{\alpha_{1}}^{\epsilon_{1}} \Gamma_{\beta_{1}}^{-\epsilon_{1}} \Gamma_{\alpha_{2}}^{\epsilon_{2}} \Gamma_{\beta_{2}}^{-\epsilon_{2}} \\
& \times\left[\left\langle T_{C} \kappa_{\alpha_{1}}^{\epsilon_{1}}\left(t^{\eta}\right) \kappa_{\beta_{1}}^{-\epsilon_{1}}\left(t^{\eta_{1}}\right) \tilde{\kappa}_{\alpha_{2}}\left(0^{-\eta}\right) \tilde{\kappa}_{\beta_{2}}^{-\epsilon_{2}}\left(0^{\eta_{2}}\right)\right\rangle-\left\langle T_{C} \kappa_{\alpha_{1}}^{\epsilon_{1}}\left(t^{\eta}\right) \kappa_{\beta_{1}}^{-\epsilon_{1}}\left(t^{\eta_{1}}\right)\right\rangle\left\langle T_{C} \tilde{\kappa}_{\alpha_{2}}^{\epsilon_{2}}\left(0^{-\eta}\right) \tilde{\kappa}_{\beta_{2}}^{-\epsilon_{2}}\left(0^{\eta_{2}}\right)\right\rangle\right] \\
& \times \eta_{1} \eta_{2} \int_{-\infty}^{\infty} e^{i \epsilon_{2} \omega_{2}\left(-\tau_{2}\right)} G_{-\eta \eta_{2}}^{v_{2} ; \eta_{1 L}}\left(\tilde{x}_{\alpha_{2}}-\tilde{x}_{\beta_{2}},-\tau_{2}\right) d \tau_{2} \int_{-\infty}^{\infty} d \tau_{1} e^{i \epsilon_{1} \omega_{1}\left(-\tau_{1}\right)} G_{\eta \eta_{1}}^{v_{11} ; v_{1 L}}\left(x_{\alpha_{1}}-x_{\beta_{1}}, t-\tau_{1}\right) .
\end{aligned}
$$

In the case where $x_{1}<x_{2}<\tilde{x}_{1}<\tilde{x}_{2}$, using the formula for the Klein factor expression found earlier, we find that the difference of Klein factor correlation functions vanishes, so that $S_{12}(t) \rightarrow 0$ for $|t| \gg 1 / T$.

Now, we focus on the case $x_{1}<\tilde{x}_{1}<x_{2}<\tilde{x}_{2}$. In this case, for $t>0$,

$$
\begin{aligned}
S_{12}(t>0)= & \frac{1}{2} \frac{1}{m^{2}} \sum_{\eta, \eta_{1}, \eta_{2} \epsilon_{1}, \epsilon_{2}= \pm \alpha_{1}, \beta_{1}, \alpha_{2}, \beta_{2}} \epsilon_{1} \epsilon_{2} \Gamma_{\alpha_{1}}^{\epsilon_{1}} \Gamma_{\beta_{1}}^{-\epsilon_{1}} \tilde{\Gamma}_{\alpha_{2}}^{\epsilon_{2}} \tilde{\Gamma}_{\beta_{2}}^{-\epsilon_{2}}\left[e^{-i\left(\epsilon_{1} \epsilon_{2} / m\right)\left(\alpha_{1}-\beta_{1}\right) \pi\left[\eta \delta_{\alpha_{2} 1}+\eta_{2} \delta_{\beta_{2} 1}\right]}-1\right] \\
& \times \eta_{1} \eta_{2} \int_{-\infty}^{\infty} e^{i \epsilon_{2} \omega_{2}\left(-\tau_{2}\right)} G_{-\eta \eta_{2}}^{v_{2 R} ; v_{1 L}}\left(\tilde{x}_{\alpha_{2}}-\tilde{x}_{\beta_{2}},-\tau_{2}\right) d \tau_{2} \int_{-\infty}^{\infty} d \tau_{1} e^{i \epsilon_{1} \omega_{1}\left(-\tau_{1}\right)} G_{\eta \eta_{1}}^{v_{1 R} ; v_{1 L}}\left(x_{\alpha_{1}}-x_{\beta_{1}}, t-\tau_{1}\right) .
\end{aligned}
$$

In what follows, we assume that the edge velocities are the same on the left and right edges, and in the two layers, which we set to unity: $v_{1 L}=v_{1 R}=v_{2 R}=1$. 
Now, let us define

$$
\begin{aligned}
A_{\eta_{1} \eta_{2}}^{\omega}(x)= & \int_{-\infty}^{\infty} e^{i \omega \tau} d \tau G_{\eta_{1} \eta_{2}}(x, \tau) \\
= & \int_{-\infty}^{\infty} d t e^{i \omega t} e^{-i(\pi / 2 m) \chi_{\eta_{1} \eta_{2}}(t)[\operatorname{sgn}(t+x)+\operatorname{sgn}(t-x)]} \\
& \times\left(\frac{(\pi T)^{2}}{\sinh [\pi T|t+x|] \sinh [\pi T|t-x|]}\right)^{1 / m} .
\end{aligned}
$$

Note that this satisfies

$$
\begin{aligned}
A_{+-}^{\omega}(x) & =A_{-+}^{-\omega}(x), \quad A_{\eta_{1} \eta_{2}}^{\omega}(x)=A_{\eta_{1} \eta_{2}}^{\omega}(-x), \\
A_{++}^{\omega}(x)+A_{--}^{\omega}(x) & =A_{+-}^{\omega}(x)+A_{-+}^{\omega}(x) .
\end{aligned}
$$

Then, we can write the noise as a simple expression:

$$
\begin{aligned}
S_{12}(t>0)= & \frac{1}{2} \frac{1}{m^{2}} \sum_{\eta, \eta_{1}, \eta_{2} \epsilon_{1}, \epsilon_{2}= \pm \alpha_{1}, \beta_{1}, \alpha_{2}, \beta_{2}} \epsilon_{1} \epsilon_{2} \eta_{1} \eta_{2}\left|\Gamma_{\alpha_{1}} \Gamma_{\beta_{1}} \tilde{\Gamma}_{\alpha_{2}} \tilde{\Gamma}_{\beta_{2}}\right| e^{i \epsilon_{1} \varphi_{\alpha_{1} \beta_{1}}+i \epsilon_{2} \tilde{\varphi}_{\alpha_{2} \beta_{2}}} \\
& \times\left[e^{-i\left(\epsilon_{1} \epsilon_{2} / m\right)\left(\alpha_{1}-\beta_{1}\right) \pi\left[\eta \delta_{\alpha_{2} 1}+\eta_{2} \delta_{\beta_{2} 1}\right]}-1\right] A_{\eta \eta_{1}}^{\epsilon_{1} \omega_{1}}\left(x_{\alpha_{1} \beta_{1}}\right) A_{-\eta \eta_{2}}^{\epsilon_{2} \omega_{2}}\left(\tilde{x}_{\alpha_{2} \beta_{2}}\right)
\end{aligned}
$$

and

$$
\begin{aligned}
S_{12}(t<0)= & \frac{1}{2} \frac{1}{m^{2}} \sum_{\eta, \eta_{1}, \eta_{2} \epsilon_{1}, \epsilon_{2}= \pm \alpha_{1}, \beta_{1}, \alpha_{2}, \beta_{2}} \epsilon_{1} \epsilon_{2} \eta_{1} \eta_{2}\left|\Gamma_{\alpha_{1}} \Gamma_{\beta_{1}} \tilde{\Gamma}_{\alpha_{2}} \tilde{\Gamma}_{\beta_{2}}\right| e^{i \epsilon_{1} \varphi_{\alpha_{1} \beta_{1}}+i \epsilon_{2} \tilde{\varphi}_{\alpha_{2} \beta_{2}}} \\
& \times\left[e^{-i\left(\epsilon_{1} \epsilon_{2} / m\right)\left(\alpha_{2}-\beta_{2}\right) \pi\left[-\eta \delta_{\alpha_{1}}+\eta_{1} \delta_{\beta_{1}}\right]}-1\right] A_{\eta \eta_{1}}^{\epsilon_{1} \omega_{1}}\left(x_{\alpha_{1} \beta_{1}}\right) A_{-\eta \eta_{2}}^{\epsilon_{2} \omega_{2}}\left(\tilde{x}_{\alpha_{2} \beta_{2}}\right) .
\end{aligned}
$$

Now, we try to make the above expressions more explicit. We start with the $t>0$ case. Summing over $\alpha_{1}$ and $\beta_{1}$ gives

$$
\begin{aligned}
S_{12}(t>0)= & \frac{1}{2} \frac{1}{m^{2}}\left|\Gamma_{1} \Gamma_{2}\right| \sum_{\eta, \eta_{1}, \eta_{2} \epsilon_{1}, \epsilon_{2}= \pm} \sum_{\alpha_{2}, \beta_{2}} \epsilon_{1} \epsilon_{2}\left|\tilde{\Gamma}_{\alpha_{2}} \tilde{\Gamma}_{\beta_{2}}\right| e^{i \epsilon_{1} \varphi_{12}+i \epsilon_{2} \tilde{\varphi}_{\alpha_{2} \beta_{2}}}\left(e^{i \epsilon_{1} \epsilon_{2}(\pi / m)\left(\eta \delta_{\alpha_{2}}+\eta_{2} \delta_{\beta_{2}}\right)}-1\right) \\
& \times \eta_{1} \eta_{2} A_{-\eta \eta_{2}}^{\epsilon_{2} \omega_{2}}\left(\tilde{x}_{12}\right)\left[A_{\eta \eta_{1}}^{\epsilon_{1} \omega_{1}}\left(x_{12}\right)-A_{\eta \eta_{1}}^{-\epsilon_{1} \omega_{1}}\left(x_{12}\right)\right] .
\end{aligned}
$$

For $S_{12}(t<0)$, we perform the sum over $\alpha_{2}, \beta_{2}$ to get

$$
\begin{aligned}
S_{12}(t<0)= & \frac{1}{2} \frac{1}{m^{2}} \sum_{\eta, \eta_{1}, \eta_{2} \epsilon_{1}, \epsilon_{2}= \pm} \sum_{\alpha_{1}, \beta_{1}} \epsilon_{1} \epsilon_{2} \eta_{1} \eta_{2}\left|\Gamma_{\alpha_{1}} \Gamma_{\beta_{1}} \tilde{\Gamma}_{1} \tilde{\Gamma}_{2}\right| e^{i \epsilon_{1} \varphi_{\alpha_{1} \beta_{1}}+i \epsilon_{2} \tilde{\varphi}_{12}} \\
& \times\left[e^{i\left(\epsilon_{1} \epsilon_{2} / m\right) \pi\left[-\eta \delta_{\alpha_{1} 2}+\eta_{1} \delta_{\beta_{1}}\right]}-1\right] A_{\eta \eta_{1}}^{\epsilon_{1} \omega_{1}}\left(x_{\alpha_{1} \beta_{1}}\right)\left[A_{-\eta \eta_{2}}^{\epsilon_{2} \omega_{2}}\left(\tilde{x}_{12}\right)-A_{-\eta \eta_{2}}^{-\epsilon_{2} \omega_{2}}\left(\tilde{x}_{12}\right)\right] .
\end{aligned}
$$

This can be written as the sum of two terms:

$$
S_{12}(t>0)=S_{12}^{1}(t>0)+S_{12}^{2}(t>0)
$$

where $S_{12}^{1}(t>0)$ contains the terms where $\alpha_{2} \neq \beta_{2}$ in the above sum. $S_{12}^{2}(t>0)$ contains terms in the sum [Eq. (D49)], where $\alpha_{2}=\beta_{2}$. Similarly, we write

$$
S_{12}(t<0)=S_{12}^{1}(t<0)+S_{12}^{2}(t<0)
$$

where $S_{12}^{1}(t<0)$ contains terms in the sum [Eq. (D50)], where $\alpha_{1} \neq \beta_{1}$, while $S_{12}^{2}(t<0)$ contains terms in the sum where $\alpha_{1}=\beta_{1}$. After simplifying, we find

$$
\begin{aligned}
S_{12}^{1}(t>0)= & \frac{(\pi T)^{4 / m-2}}{m^{2}}\left|\Gamma_{1} \Gamma_{2} \tilde{\Gamma}_{1} \tilde{\Gamma}_{2}\right| \\
& \times S_{12}^{(2)}\left(\bar{\omega}_{1}, \bar{\omega}_{2}, \pi T x_{12}, \pi T \tilde{x}_{12}, \tilde{\varphi}_{12}, \varphi_{12}\right), \\
S_{12}^{2}(t>0)= & \frac{(\pi T)^{4 / m-2}}{m^{2}}\left|\tilde{\Gamma}_{1}\right|^{2}\left|\Gamma_{1} \Gamma_{2}\right| \\
& \times S_{12}^{(1)}\left(\bar{\omega}_{1}, \bar{\omega}_{2}, \pi T x_{12}, \varphi_{12}\right), \\
S_{12}^{1}(t<0)= & \frac{(\pi T)^{4 / m-2}}{m^{2}}\left|\Gamma_{1} \Gamma_{2} \tilde{\Gamma}_{1} \tilde{\Gamma}_{2}\right| \\
& \times S_{12}^{(2)}\left(\bar{\omega}_{2}, \bar{\omega}_{1}, \pi T \tilde{x}_{12}, \pi T x_{12}, \varphi_{12}, \tilde{\varphi}_{12}\right), \\
S_{12}^{2}(t<0)= & \frac{(\pi T)^{4 / m-2}}{m^{2}}\left|\Gamma_{1}\right|^{2}\left|\tilde{\Gamma}_{1} \tilde{\Gamma}_{2}\right| \\
& \times S_{12}^{(1)}\left(\bar{\omega}_{2}, \bar{\omega}_{1}, \pi T \tilde{x}_{12}, \tilde{\varphi}_{12}\right),
\end{aligned}
$$

where $S_{12}^{(i)}$ are presented in Eq. (D16). 


\section{APPENDIX E: TOPOLOGICALLY PROTECTED GATES AND BRAIDING OF PARAFERMION ZERO MODES}

In this section, we briefly describe one way to perform topologically protected operations on the qubits proposed in the main text, adapting the results of Ref. [15]. The main idea is that the non-Abelian defects can be effectively braided using a $T$-junction setup (see Fig. 8). This is similar to the proposal for braiding Majorana zero modes in 1D topological superconductors [37]. Figure 8 illustrates one specific protocol for braiding a pair of genons, which requires tuning the various gates on and off adiabatically in the pattern indicated.

Suppose that there are $n$ pairs of genons in the bilayer system, with a 1/3 Laughlin FQH state in each layer. Then, each pair of genons can be associated with three topologically degenerate states, for a total of $3^{n}$ states. We can label these different topological states as $\left|\left\{q_{i}\right\}\right\rangle, i=1, \ldots, n$, and $q_{i}=0,1,2$, where $q_{i}$ labels the state associated with the $(2 i-1)$ and $2 i$ th genon. According to Ref. [15], the braiding of genons $(2 i-1)$ and $2 i$ leads to a unitary operation $U_{2 i-1,2 i}$ on $\left|\left\{q_{i}\right\}\right\rangle$, given by

$$
U_{2 i-1,2 i}\left|\left\{q_{i}\right\}\right\rangle=e^{i \theta} e^{i \pi i\left(q_{i}-k\right)^{2} / m}\left|\left\{q_{i}\right\}\right\rangle,
$$

where $e^{i \theta}$ is an overall nontopological phase and $k$ is an integer that depends on details of the braiding protocol, but which is robust to perturbations [15,16,17]. The unitary operation $U_{2 i, 2 i+1}$, associated with braiding genons $2 i$ and $2 i+1$, does not commute with $U_{2 i-1,2 i}$ and can be obtained from the considerations of Ref. [15].
As was shown in Ref. [16], these braiding operations lead to a topologically protected unitary set of gates that is somewhat more computationally powerful than those available for Majorana zero modes. Specifically, the braiding operations of Majorana zero modes alone cannot produce a 2-qubit entangling gate. In order to achieve this, one must perform a joint fermion parity measurement on four Majorana zero modes, which may be much more complicated than the braiding. In contrast, the genons described here can, in general, realize a 2-qubit entangling gate through braiding operations alone, without requiring a joint measurement on the state of multiple genons. This can be done using the following sequence of gates [16]:

$$
C P=\left(U_{23} U_{12} U_{34} U_{23}\right)^{2} .
$$

When acting on two pairs of genons, this gives

$$
C P\left|q_{1}, q_{2}\right\rangle=e^{-\left(4 \pi i q_{1} q_{2} / 3\right)}\left|q_{1}, q_{2}\right\rangle,
$$

which can successfully entangle the state of the two pairs of genons. Here, for simplicity we have set the integer $k=0$.

Despite this improvement, the braiding of genons using Abelian FQH states, like those of Majorana fermion zero modes, is not sufficient for universal topological quantum computation: Both are lacking the single-qubit $\pi / 8$ phase gate. However, realizing genons is still helpful for achieving universal topological quantum computation if we consider genons in more general systems. As was described in Ref. [15], in a double-layer system with each layer nonAbelian but not universal for TQC, braiding of genons can be used to perform universal TQC. The simplest example is the bilayer Ising topological phase, in which case the genon (a)
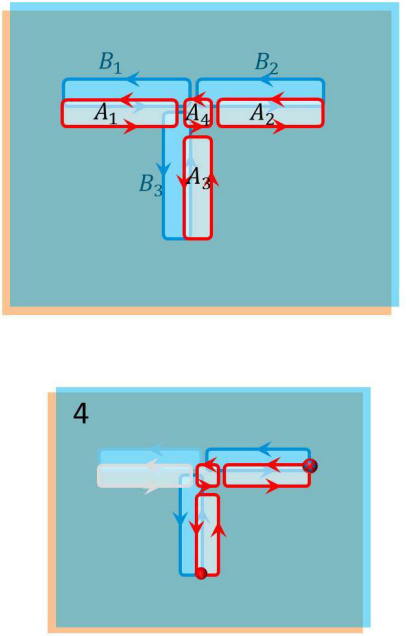

(b)
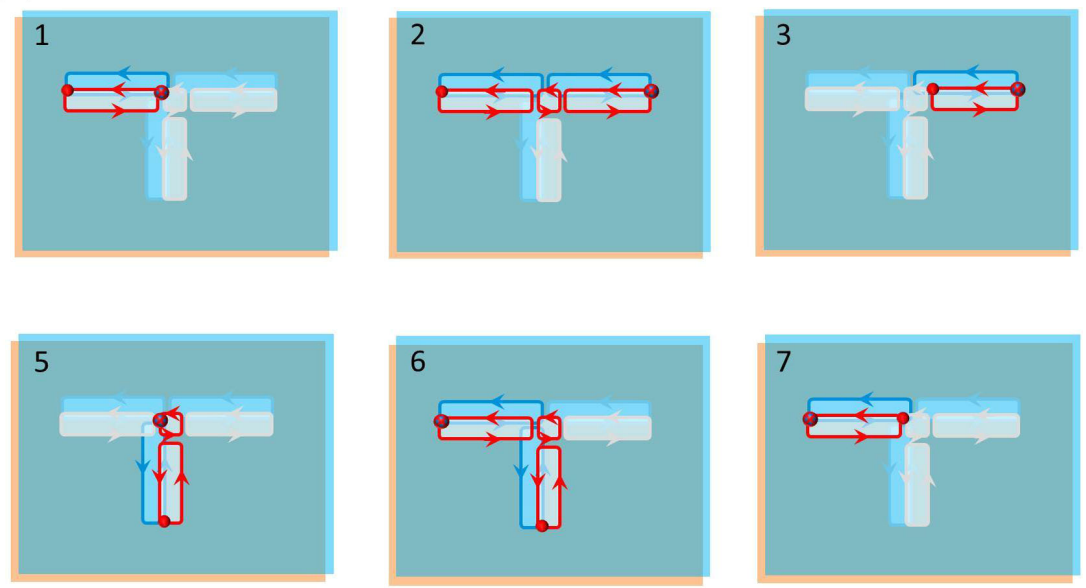

FIG. 8. (a) $T$-junction gate configuration that can be used to braid two nearby zero modes. There are four top gates, labeled $A_{1}, \ldots, A_{4}$, and three bottom gates, labeled $B_{1}, B_{2}, B_{3}$. (b) Protocol for braiding two zero modes, which are marked by the circles. One of them is marked with a red " $x$ " in order to track its position. Gates with edge states shown in gray are turned off, those with the edge modes shown in red (top layer) or blue (bottom layer) are on. Adiabatically turning the gates on and off in the pattern shown in (1)-(7) effectively braids the zero modes. 
braiding realizes the $\pi / 8$ phase gate that cannot be realized by braiding non-Abelian anyons in each layer. We do not review this proposal here, and instead refer the reader to Ref. [15] for details.

[1] C. Nayak, S. H. Simon, A. Stern, M. Freedman, and S. Das Sarma, Non-Abelian Anyons and Topological Quantum Computation, Rev. Mod. Phys. 80, 1083 (2008).

[2] X.-G. Wen, Quantum Field Theory of Many-Body Systems (Oxford University Press, Oxford, England, 2004).

[3] J. Alicea, New Directions in the Pursuit of Majorana Fermions in Solid State Systems, Rep. Prog. Phys. 75, 076501 (2012).

[4] G. Moore and N. Read, Nonabelions in the Fractional Quantum Hall Effect, Nucl. Phys. B360, 362 (1991).

[5] X.-G. Wen, Non-Abelian Statistics in the Fractional Quantum Hall States, Phys. Rev. Lett. 66, 802 (1991).

[6] R. L. Willett, L. N. Pfeiffer, and K. W. West, Measurement of Filling Factor 5/2 Quasiparticle Interference with Observation of Charge e/4 and e/2 Period Oscillations, Proc. Natl. Acad. Sci. U.S.A. 106, 8853 (2009).

[7] N. Read and D. Green, Paired States of Fermions in Two Dimensions with Breaking of Parity and Time-Reversal Symmetries and the Fractional Quantum Hall Effect, Phys. Rev. B 61, 10267 (2000).

[8] J. D. Sau, R. M. Lutchyn, S. Tewari, and S. Das Sarma, Generic New Platform for Topological Quantum Computation Using Semiconductor Heterostructures, Phys. Rev. Lett. 104, 040502 (2010).

[9] J. D. Sau, S. Tewari, R. M. Lutchyn, T. D. Stanescu, and S. Das Sarma, Non-Abelian Quantum Order in Spin-OrbitCoupled Semiconductors: Search for Topological Majorana Particles in Solid-State Systems, Phys. Rev. B 82, 214509 (2010).

[10] Y. Oreg, G. Refael, and F. von Oppen, Helical Liquids and Majorana Bound States in Quantum Wires, Phys. Rev. Lett. 105, 177002 (2010).

[11] R. M. Lutchyn, J. D. Sau, and S. Das Sarma, Majorana Fermions and a Topological Phase Transition in Semiconductor-Superconductor Heterostructures, Phys. Rev. Lett. 105, 077001 (2010).

[12] V. Mourik, K. Zuo, S. M. Frolov, S. R. Plissard, E. P. A. M. Bakkers, and L. P. Kouwenhoven, Signatures of Majorana Fermions in Hybrid Superconductor-Semiconductor Nanowire Devices, Science 336, 1003 (2012).

[13] L. P. Rokhinson, X. Liu, and J. K. Furdyna, The Fractional a.c. Josephson Effect in a Semiconductor-Superconductor Nanowire as a Signature of Majorana Particles, Nat. Phys. 8, 795 (2012).

[14] M. Barkeshli and X.-L. Qi, Topological Nematic States and Non-Abelian Lattice Dislocations, Phys. Rev. X 2, 031013 (2012).

[15] M. Barkeshli, C.-M. Jian, and X.-L. Qi, Twist Defects and Projective Non-Abelian Braiding Statistics, Phys. Rev. B 87, 045130 (2013).

[16] D. J. Clarke, J. Alicea, and K. Shtengel, Exotic Non-Abelian Anyons from Conventional Fractional Quantum Hall States, Nat. Commun. 4, 1348 (2013).
[17] N. H. Lindner, E. Berg, G. Refael, and A. Stern, Fractionalizing Majorana Fermions: Non-Abelian Satistics on the Edges of Abelian Quantum Hall States, Phys. Rev. X 2 , 041002 (2012).

[18] M. Cheng, Superconducting Proximity Effect on the Edge of Fractional Topological Insulators, Phys. Rev. B 86, 195126 (2012).

[19] G. S. Boebinger, H. W. Jiang, L. N. Pfeiffer, and K. W. West, Magnetic-Field-Driven Destruction of Quantum Hall States in a Double Quantum Well, Phys. Rev. Lett. 64, 1793 (1990).

[20] Y. W. Suen, J. Jo, M. B. Santos, L. W. Engel, S. W. Hwang, and M. Shayegan, Missing Integral Quantum Hall Effect in a Wide Single Quantum Well, Phys. Rev. B 44, 5947 (1991).

[21] J. P. Eisenstein, G. S. Boebinger, L. N. Pfeiffer, K. W. West, and S. He, New Fractional Quantum Hall State in DoubleLayer Two-Dimensional Electron Systems, Phys. Rev. Lett. 68, 1383 (1992).

[22] T. S. Lay, T. Jungwirth, L. Smrčka, and M. Shayegan, OneComponent to Two-Component Transition of the $\nu=2 / 3$ Fractional Quantum Hall Effect in a Wide Quantum Well Induced by an In-Plane Magnetic Field, Phys. Rev. B 56, R7092 (1997).

[23] H. C. Manoharan, Y. W. Suen, T. S. Lay, M. B. Santos, and M. Shayegan, Spontaneous Interlayer Charge Transfer near the Magnetic Quantum Limit, Phys. Rev. Lett. 79, 2722 (1997).

[24] X. G. Wen, Theory of the Edge States in Fractional Quantum Hall Effects, Int. J. Mod. Phys. B 06, 1711 (1992).

[25] A. Sciambi, M. Pelliccione, S. R. Bank, A. C. Gossard, and D. Goldhaber-Gordon, Virtual Scanning Tunneling Microscopy: A Local Spectroscopic Probe of Two-Dimensional Electron Systems, Appl. Phys. Lett. 97, 132103 (2010).

[26] D. Goldhaber-Gordon (private communication).

[27] A. Sciambi, M. Pelliccione, M. P. Lilly, S. R. Bank, A. C. Gossard, L. N. Pfeiffer, K. W. West, and D. GoldhaberGordon, Vertical Field-Effect Transistor Based on WaveFunction Extension, Phys. Rev. B 84, 085301 (2011).

[28] C. L. Kane and M. P. A. Fisher, Line Junctions in the Quantum Hall Effect, Phys. Rev. B 56, 15231 (1997).

[29] X. G. Wen and Q. Niu, Ground-State Degeneracy of the Fractional Quantum Hall States in the Presence of a Random Potential and on High-Genus Riemann Surfaces, Phys. Rev. B 41, 9377 (1990).

[30] A. Vaezi, Fractional Topological Superconductor with Fractionalized Majorana Fermions, Phys. Rev. B 87, 035132 (2013).

[31] P. Fendley, Parafermionic Edge Zero Modes in Zn-Invariant Spin Chains, J. Stat. Mech. (2012) P11020.

[32] K. T. Law, P. A. Lee, and T. K. Ng, Majorana Fermion Induced Resonant Andreev Reflection, Phys. Rev. Lett. 103, 237001 (2009).

[33] L. Fidkowski, J. Alicea, N. H. Lindner, R. M. Lutchyn, and M.P. A. Fisher, Universal Transport Signatures of Majorana Fermions in Superconductor-Luttinger Liquid Junctions, Phys. Rev. B 85, 245121 (2012).

[34] P. Bonderson, A. Kitaev, and K. Shtengel, Detecting NonAbelian Statistics in the $\nu=5 / 2$ Fractional Quantum Hall State, Phys. Rev. Lett. 96, 016803 (2006).

[35] A. Stern and B. I. Halperin, Proposed Experiments to Probe the Non-Abelian $\nu=5 / 2$ Quantum Hall State, Phys. Rev. Lett. 96, 016802 (2006). 
[36] C. L. Kane, Telegraph Noise and Fractional Statistics in the Quantum Hall Effect, Phys. Rev. Lett. 90, 226802 (2003).

[37] J. Alicea, Y. Oreg, G. Refael, F. von Oppen, and M. P. A. Fisher, Non-Abelian Statistics and Topological Quantum Information Processing in 1D Wire Networks, Nat. Phys. 7, 412 (2011).

[38] A. Kitaev, Anyons in an Exactly Solved Model and Beyond, Ann. Phys. (Amsterdam) 321, 2 (2006).

[39] P. Bonderson and J. K. Slingerland, Fractional Quantum Hall Hierarchy and the Second Landau Level, Phys. Rev. B 78, 125323 (2008).
[40] P. Bonderson (private communication).

[41] C. de C. Chamon, D. E. Freed, and X. G. Wen, Tunneling and Quantum Noise in One-Dimensional Luttinger Liquids, Phys. Rev. B 51, 2363 (1995).

[42] C. de C. Chamon, D. E. Freed, S. A. Kivelson, S. L. Sondhi, and X. G. Wen, Two Point-Contact Interferometer for Quantum Hall Systems, Phys. Rev. B 55, 2331 (1997).

[43] R. Guyon, P. Devillard, T. Martin, and I. Safi, Klein Factors in Multiple Fractional Quantum Hall Edge Tunneling, Phys. Rev. B 65, 153304 (2002). 